CERN-TH/98-212

CPTH-S616.0698

LPTHE-ORSAY 98/42

ROM2F-98/21

hep-th/9807011

\title{
Supersymmetry breaking, open strings and M-theory
}

\author{
I. Antoniadis ${ }^{a, b}$, E. Dudas ${ }^{b, c}$ and A. Sagnotti ${ }^{d}$ \\ ${ }^{a}$ Centre de Physique Théorique, Ecole Polytechnique, F-91128 Palaiseau, France \\ b TH-Division, CERN, CH-1211 Geneva 23, Switzerland \\ ${ }^{c}$ LPTHE ${ }^{\dagger}$, Bât. 211, Univ. Paris-Sud, F-91405 Orsay, France \\ d Dipartimento di Fisica, Universita di Roma "Tor Vergata", INFN, Sezione di Roma, \\ Via della Ricerca Scientifica 1, 00133 Roma, Italy
}

\begin{abstract}
We study supersymmetry breaking by Scherk-Schwarz compactifications in type I string theory. While in the gravitational sector all mass splittings are proportional to a (large) compactification radius, supersymmetry remains unbroken for the massless excitations of D-branes orthogonal to the large dimension. In this sector, supersymmetry breaking can then be mediated by gravitational interactions alone, that are expected to be suppressed by powers of the Planck mass. The mechanism is non perturbative from the heterotic viewpoint and requires a compactification radius at intermediate energies of order $10^{12}-10^{14} \mathrm{GeV}$. This can also explain the value of Newton's constant if the string scale is close to the unification scale, of order $10^{16}$ $\mathrm{GeV}$.
\end{abstract}

${ }^{\star}$ Research supported in part by the EEC under TMR contract ERBFMRX-CT96-0090.

${ }^{\dagger}$ Laboratoire associé au CNRS-URA-D0063.

CERN-TH/98-212

July 1998 


\section{Introduction}

In perturbative string theory supersymmetry breaking through compactification relates the breaking scale to the size of a compact dimension. In weakly coupled heterotic strings, one thus obtains the (tree-level) relation:

$$
m_{3 / 2}=m_{1 / 2} \sim R^{-1}
$$

where $m_{3 / 2}$ and $m_{1 / 2}$ are the gravitino and gaugino masses, while $R$ is the radius of the extra dimension. Therefore, phenomenologically acceptable soft masses ask for a very large radius, in the $\mathrm{TeV}^{-1}$ region. However, since Standard Model gauge bosons feel the extra dimension, only in special models can one avoid large corrections to the gauge couplings and accommodate the existing phenomenology [1, 2]. The resulting scalar masses $m_{0}$ are then in general model dependent. In the simplest case, chiral families live on boundaries of space orthogonal to the $\mathrm{TeV}$ dimension, and thus do not feel it; they correspond to $N=1$ twisted states and satisfy $m_{0}=0$. This guarantees flavor universality, avoids potential problems with proton decay and makes model building easier and more natural.

The Scherk-Schwarz mechanism provides an elegant realization of supersymmetry breaking by compactification in field theory [3]. In the simplest case of circle compactification, it amounts to allowing the higher dimensional fields to be periodic around the circle up to an R-symmetry transformation. The Kaluza-Klein momenta of the various fields are correspondingly shifted in a way proportional to their R-charges. The R-transformations are actually restricted to discrete subgroups, as are all global internal symmetries of supergrav-

ity models, since the massive excitations lead to effective Dirac-like quantizations of the corresponding parameters. As a result, the scale of supersymmetry breaking is quantized in units of the compactification scale. Modular invariance dictates the extension of this mechanism to the full perturbative spectrum in models of oriented closed strings [4, 5, 1].

Breaking supersymmetry by compactification rather than dynamically via gaugino con- 
densation [6] has the obvious advantage of calculability. However, in the perturbative heterotic string this breaking appears to be too restrictive, and it is interesting to inquire whether other possibilities can be realized within different perturbative string descriptions.

In this work we study supersymmetry breaking by compactification in the type I theory of open and closed strings. This is closely related to the type-IIB theory of oriented closed strings [7], and can also describe the heterotic string at strong coupling [8, 9, 10]. In particular, we generalize the Scherk-Schwarz mechanism to open strings. Although the gravitino mass is still proportional to a compactification radius, we find that the gaugino mass can actually decouple. This occurs whenever in the type I theory gauge particles live on D-branes orthogonal to the large dimension. In the low-energy spectrum supersymmetry breaking then affects only the bulk to lowest order, and thus

$$
m_{3 / 2} \sim R^{-1}, \quad m_{1 / 2}=0
$$

while on the brane it is mediated by gravitational interactions, expected to be suppressed by powers of the Planck mass $M_{P}$, so that effectively $m_{1 / 2} \lesssim \mathcal{O}\left(m_{3 / 2}^{2} / M_{P}\right)$. As a result, the compactification radius could be at an intermediate scale, $R^{-1} \sim 10^{12}-10^{14} \mathrm{GeV}$. These models do not require special conditions to avoid the large coupling problem, since the large dimension is felt by matter fields only via gravitational interactions.

This scenario was suggested [11] in the context of M-theory, where supersymmetry breaking could be related to the radius of the eleventh dimension [11, 12]. In fact, as we will show in Section 4, upon compactification to 9 dimensions there is an equivalent (dual) description as a weakly coupled type $\mathrm{I}^{\prime}$ theory. Models with an intermediate scale compactification and with the breaking conditions (1.2) are certainly non-perturbative when viewed from the heterotic side. They share many properties with models of gaugino condensation, and may even provide a dual description of it if the condensation scale is the M-theory scale and the generated superpotential is of order one in Planck units.

This paper is organized as follows. In Section 2 we discuss gauge coupling unification 
in type I theories and show that it is compatible with intermediate scale compactification consistently with the supersymmetry breaking masses (1.2). In Section 3 we review the BPS spectrum in $N=1$ compactifications to nine dimensions obtained either from Mtheory on $S^{1} \times S^{1} / Z_{2}$ or from type $I$ (or type $I^{\prime}$ ) string theory on $S^{1}$. In Section 4 we discuss supersymmetry breaking by the Scherk-Schwarz mechanism in the effective field theory and in closed string theory. In Section 5 we generalize this mechanism to open strings and give explicit examples in nine dimensions. We find two distinct possibilities. The first extends to the type I string the ordinary Scherk-Schwarz compactification, and can also be obtained by duality from the perturbative $S O(32)$ heterotic string in the limit where the winding modes decouple from the gauge sector. It also defines type I theory at finite temperature, when the compactified coordinate is identified with the (euclidean) time. The second corresponds to a similar compactification on the type $\mathrm{I}^{\prime}$ side, but now the $S O(16) \times S O(16)$ gauge group lives on D8-branes orthogonal to the dimension used to break supersymmetry. As a result, supersymmetry remains unbroken in the massless gauge sector. By duality, this mechanism is equivalent to Scherk-Schwarz supersymmetry breaking along the 11th dimension on the M-theory side, and is non-perturbative from the heterotic point of view. In Section 6 we compute the one-loop cosmological constant and the scalar masses for the two models. In the second case, the corrections to both quantities from open-string loops are exponentially suppressed in the large radius limit. This result agrees with the field theory expectation that, in the absence of quadratic divergences, supersymmetry breaking mediated by gravitational interactions should be suppressed by powers of the Planck mass. In Section 7 we generalize the construction to six dimensions and present an explicit chiral model with broken $(1,0)$ supersymmetry. 


\section{Unification and supersymmetry breaking in open strings and in M-theory}

In this Section, we consider unification constraints in type I and type I' models and corresponding scenarios of intermediate scale supersymmetry breaking via compactification along a certain direction in the compact space. In nine-dimensional models, this direction can be identified with the eleventh dimension of M-theory, as we shall see in detail in the next Section.

In heterotic models, the ten-dimensional string coupling $\lambda_{H}$ and the string scale $M_{H}=$ $\left(\alpha^{\prime}\right)^{-1 / 2}$ are expressed in terms of four dimensional parameters as

$$
\lambda_{H}=\frac{\left(\alpha_{G}\right)^{2}}{8 \sqrt{2}} V^{1 / 2} M_{P}^{3}, \quad M_{H}=\left(\frac{\alpha_{G}}{8}\right)^{1 / 2} M_{P}
$$

where $(2 \pi)^{6} V$ is the volume of the six-dimensional internal manifold, $\alpha_{G} \sim \frac{1}{25}$ is the gauge coupling at the unification scale and $M_{P}=G_{N}^{-1 / 2}$ is the Planck mass. The corresponding relations for the open superstring can be deduced using the duality between the heterotic $\mathrm{SO}(32)$ and the type I string in $10 \mathrm{~d}[8]$ :

$$
\lambda_{I}=\frac{1}{\lambda_{H}}, \quad M_{I}=M_{H} \lambda_{H}^{-1 / 2}
$$

Thus

$$
\lambda_{I}=\frac{8 \sqrt{2}}{\left(\alpha_{G}\right)^{2}} V^{-1 / 2} M_{P}^{-3}, \quad M_{I}=\left(\frac{\sqrt{2}}{\alpha_{G} M_{P}}\right)^{1 / 2} V^{-1 / 4},
$$

where $\alpha_{G}$ is the coupling for nine-brane gauge fields. The unification scale $M_{G U T}$ is fixed by the fundamental mass scale $M_{I}$, and therefore in the following we take $M_{I} \sim 3 \times 10^{16} \mathrm{GeV}$.

In the simplest case of an isotropic compact space with $V=r^{6} M_{I}^{-6}$, the natural values of the dimensionless radius $r$ are of order one. In particular, $r=1$ corresponds to the self-dual point of circle compactification. In this case $M_{I}=\left(\alpha_{G} / \sqrt{2}\right) r^{3} M_{P}$, that requires $r \sim \frac{1}{6}$, and all the corresponding radii are of the order of the unification scale. 
The situation changes drastically for anisotropic compactifications. For instance, if one radius $R_{I}$ is large and five others are of order $r M_{I}^{-1}\left(V=r^{5} M_{I}^{-5} R_{I}\right)$, eq. (2.3) leads to

$$
R_{I}^{-1}=\left(\frac{\alpha_{G}}{\sqrt{2}}\right)^{2} r^{5} \frac{M_{P}^{2}}{M_{I}}, \quad \lambda_{I}=\frac{8}{\alpha_{G}}\left(\frac{M_{I}}{M_{P}}\right)^{2} .
$$

Since $R_{I}^{-1}>>M_{I}$, it is convenient to perform a T-duality along the $R_{I}$ direction to turn to the type I' description. The corresponding duality relations are

$$
M_{I^{\prime}}=M_{I}, \quad R_{I^{\prime}}=M_{I}^{-2} / R_{I}, \quad \lambda_{I^{\prime}}=\lambda_{I} /\left(R_{I} M_{I}\right)
$$

and therefore

$$
R_{I^{\prime}}^{-1}=\left(\frac{\sqrt{2}}{\alpha_{G}}\right)^{2} r^{-5} \frac{M_{I}^{3}}{M_{P}^{2}}, \quad \lambda_{I^{\prime}}=4 \alpha_{G} r^{5} .
$$

Interestingly, these relations are similar to those obtained by Horava and Witten, if $R_{I^{\prime}}$ is identified with the radius of the eleventh dimension in $\mathrm{M}$ theory [10, 11]. The relation between the corresponding BPS spectra will be discussed in detail in the following Section. Actually, eq. (2.6) motivated the suggestion 11] that the Scherk-Schwarz mechanism along the eleventh dimension of M-theory can describe gaugino condensation with a condensation scale $\Lambda \sim M_{I}$, since in the latter case $m_{3 / 2} \sim \Lambda^{3} / M_{P}^{2}$. In particular, with $r \sim 1$ eq. (2.6) gives $m_{3 / 2} \sim R_{I^{\prime}}^{-1} \sim 10^{13} \mathrm{GeV}$. In the following Sections we will see that, in a class of models, charged matter fields associated to branes orthogonal to the dimension of radius $R_{I^{\prime}}$ feel the breaking of supersymmetry only through Planck suppressed radiative corrections. Therefore, this scenario is of potential interest for phenomenology.

If two radii $R_{I}$ are large and the other four have natural values $r M_{I}^{-1}$, one finds

$$
\begin{array}{ll}
R_{I}^{-1}=\frac{\alpha_{G} r^{2}}{\sqrt{2}} M_{P}, & \lambda_{I}=\frac{8}{\alpha_{G}}\left(\frac{M_{I}}{M_{P}}\right)^{2}, \\
R_{I^{\prime}}^{-1}=\frac{\sqrt{2}}{\alpha_{G} r^{2}} \frac{M_{I}^{2}}{M_{P}}, & \lambda_{I^{\prime}}=4 \alpha_{G} r^{4} .
\end{array}
$$

Once more, it is convenient to use the type $\mathrm{I}^{\prime}$ description, but now $R_{I^{\prime}}^{-1}$ is raised by roughly one order of magnitude. While this may still be acceptable for phenomenology, additional 
large internal radii have the effect of moving the intermediate scale further toward the unification scale.

\section{BPS spectra in nine dimensions}

The basic features of the breaking mechanism are already visible in the compactification from ten to nine dimensions. Therefore, in this Section we restrict our attention to the type I theory compactified on a circle of radius $R$. T-duality relates this analysis to the Horava-Witten reduction of $\mathrm{M}$ theory, and thus provides an explicit description of the Scherk-Schwarz breaking along the eleventh dimension. The compactification of M-theory on $S^{1} \times S^{1} / Z_{2}$ (with radii $R_{10}$ and $R_{11}$, respectively) admits two different interpretations [10]:

1. as M-theory on $S^{1} / Z_{2} \times S^{1}$, that according to [10] describes the $E_{8} \times E_{8}$ heterotic string of coupling $\lambda_{E_{8} \times E_{8}}=\left(R_{11} M_{11}\right)^{3 / 2}$, compactified on a circle $S^{1}$ of radius $R_{10}\left(R_{11} M_{11}\right)^{1 / 2}$. In this case, a Wilson line must be added, and the theory is in a vacuum with an unbroken $S O(16) \times S O(16)$ gauge group.

2. as M-theory on $S^{1} \times S^{1} / Z_{2}$, that according to [8] describes the IIA theory of coupling $\lambda_{I I A}=\left(R_{10} M_{11}\right)^{3 / 2}$, compactified further on the $S^{1} / Z_{2}$ orientifold of radius $R_{11}\left(R_{10} M_{11}\right)^{1 / 2}$. The result is the type-I' theory, T-dual (with respect to the eleventh coordinate) to the type I theory (in its $S O(16) \times S O(16)$ vacuum), with coupling $\lambda_{I}=R_{10} / R_{11}$, compactified on a circle of radius $M_{I}^{-2} /\left(R_{11}\left(R_{10} M_{11}\right)^{1 / 2}\right)$. In the Mtheory regime $\left(R_{11}>>R_{10}\right)$, the type I and type $\mathrm{I}^{\prime}$ theories can both be weakly coupled, and can consequently be treated as perturbative strings.

It is particularly instructive to translate in type I or heterotic language the masses of the BPS states of M-theory [10]. Consider first the Kaluza-Klein states of the supergravity multiplet on $T^{2}=S^{1} \times S^{1}$, together with the wrapping modes of the membrane around 
the torus. Their masses are

$$
\mathcal{M}^{2}=\frac{l^{2}}{R_{11}^{2}}+\frac{m^{2}}{R_{10}^{2}}+n^{2} R_{10}^{2} R_{11}^{2} M_{11}^{6}
$$

where $(l, m, n)$ is a triplet of integers labelling the corresponding charges. In the effective field theory, supersymmetry breaking along the eleventh dimension results from shifts [3]

$$
l \rightarrow l+\omega
$$

where $\omega$ is the R-charge of the corresponding state. Ordinary Kaluza-Klein excitations in the tenth direction with charges $m$ do not feel the breaking, while the wrapping modes labelled by $n$ in principle do.

In type-I and type-I' units, the masses of the states (3.1) are

$$
\mathcal{M}_{I}^{2}=l^{2} R_{I}^{2} M_{I}^{4}+\frac{m^{2} R_{I}^{2} M_{I}^{4}}{\lambda_{I}{ }^{2}}+\frac{n^{2}}{R_{I}^{2}}, \quad \mathcal{M}_{I^{\prime}}^{2}=\frac{l^{2}}{R_{I^{\prime}}^{2}}+\frac{m^{2} M_{I}^{2}}{\lambda_{I^{\prime}}{ }^{2}}+n^{2} R_{I^{\prime}}^{2} M_{I}^{4} .
$$

Therefore, from the type $\mathrm{I}^{\prime}$ viewpoint the breaking along the eleventh dimension gives Kaluza-Klein type masses proportional to $R_{I^{\prime}}^{-1}$, and supersymmetry is restored in the $R_{I^{\prime}} \rightarrow \infty$ limit. This is the string generalization of the field-theoretical Scherk-Schwarz mechanism. In the zero-winding sector $(n=0)$, it reduces to the ordinary Scherk-Schwarz breaking effected by the momentum shift (3.2), while the extension to the whole (perturbative) closed-string spectrum is determined by modular invariance. On the other hand, from the type I viewpoint, this breaking gives winding type masses proportional to $R_{I} M_{I}^{2}$, and supersymmetry is restored in the $R_{I} \rightarrow 0$ limit. As a result, there is no field-theoretical description corresponding to this case.

In a similar fashion, in $E_{8} \times E_{8}$ and $S O(32)$ heterotic units the states (3.1) have masses

$$
\mathcal{M}_{E_{8}}^{2}=\frac{l^{2} M_{H}^{2}}{\lambda_{E_{8}}{ }^{2}}+\frac{m^{2}}{R_{E_{8}}^{2}}+n^{2} R_{E_{8}}^{2} M_{H}^{4}, \quad \mathcal{M}_{H}^{2}=l^{2} \frac{R_{H}^{2} M_{H}^{4}}{\lambda_{H}{ }^{2}}+m^{2} R_{H}^{2} M_{H}^{4}+\frac{n^{2}}{R_{H}^{2}}
$$

Notice that in both heterotic theories the shift $l \rightarrow l+\omega$ gives masses of non-perturbative type, and thus the breaking along the eleventh dimension is a genuinely non-perturbative 
phenomenon. Similarly, the perturbative heterotic states labelled by $m$ turn into nonperturbative ones in the type I or type I' descriptions.

There are also twisted M-theory states associated to the fixed points of $S^{1} / Z_{2}$, that are charged under the gauge group. These include ordinary momentum excitations in the tenth direction and membrane wrappings in the full internal space, for which

$$
\mathcal{M}^{2}=\frac{\tilde{m}^{2}}{R_{10}^{2}}+\tilde{n}^{2} R_{10}^{2} R_{11}^{2} M_{11}^{6}
$$

As before, the charged Kaluza-Klein states labelled by $\tilde{m}$ do not feel the Scherk-Schwarz breaking along the eleventh coordinate, but the wrapping modes $\tilde{n}$ in principle do. In type I and type I' units, their masses become

$$
\mathcal{M}_{I}^{2}=\frac{\tilde{m}^{2} R_{I}^{2} M_{I}^{4}}{\lambda_{I}^{2}}+\frac{\tilde{n}^{2}}{R_{I}^{2}}, \quad \mathcal{M}_{I^{\prime}}^{2}=\frac{\tilde{m}^{2} M_{I}^{2}}{\lambda_{I^{\prime}}^{2}}+\tilde{n}^{2} R_{I^{\prime}}^{2} M_{I}^{4}
$$

and the wrapping modes are thus perturbative open string states. In $E_{8} \times E_{8}$ and $S O(32)$ heterotic units, the masses of the charged states are

$$
\mathcal{M}_{E_{8}}^{2}=\frac{\tilde{m}^{2}}{R_{E_{8}}^{2}}+\tilde{n}^{2} R_{E_{8}}^{2} M_{H}^{4}, \quad \mathcal{M}_{H}^{2}=\tilde{m}^{2} R_{H}^{2} M_{H}^{4}+\frac{\tilde{n}^{2}}{R_{H}^{2}} .
$$

From the $E_{8} \times E_{8}$ viewpoint, the breaking along the eleventh dimension can affect the windings, that can be seen only at the string level. Thus, from the field theory (KaluzaKlein) point of view, the charged states are unaffected.

This mechanism can be contrasted with the ordinary Scherk-Schwarz mechanism of perturbative heterotic strings, that amounts to shifts of $n$ and $\tilde{n}$ (or $m$ and $\tilde{m}$ ) for the $S O(32)$ (or $\left.E_{8} \times E_{8}\right)$ model (see eqs. (3.4) and (3.7)). The perturbative states labelled by $n$ and $\tilde{n}$ have counterparts in the type I theory that reflect the perturbative ninedimensional duality between the two models (see eqs. (3.3) and (3.6)). This is effective if the string coupling $\lambda_{I}$ is small and $R_{I}$ is large. 


\section{Scherk-Schwarz mechanism for oriented closed strings}

In order to make the previous analysis more explicit, in this Section we review the basic features of the breaking mechanism in models of oriented closed strings, following [5]. In the next Section we shall extend these results to the open descendants, describing corresponding breaking patterns in type I (or type I') models. The Scherk-Schwarz mechanism results from a discrete deformation of the type-IIB partition function compatible with modular invariance [4, 5]. The starting point of our discussion is thus the partition function for the circle reduction of the type-IIB string,

$$
\mathcal{T}=\frac{\tau_{2}^{-7 / 2}}{|\eta(\tau)|^{22}} \sum_{m, n=-\infty}^{\infty} Z_{m, n}(\tau, \bar{\tau})\left|\sum_{\substack{\text { spin } \\
\text { structures }}} C\left(\begin{array}{l}
\mathbf{a} \\
\mathbf{b}
\end{array}\right) \theta^{4}\left(\begin{array}{l}
\mathbf{a} \\
\mathbf{b}
\end{array}\right)(\tau)\right|^{2},
$$

where $(m, n)$ is a pair of integers labelling the Kaluza-Klein momentum and the winding number in the compactified dimension, and the $C$ 's are phases specifying the GSO projection and depending on the spin structures labelled by the pair $(\mathbf{a}, \mathbf{b})$. The $\theta$ 's are Jacobi theta functions, $\eta$ is the Dedekind function, $\tau=\tau_{1}+i \tau_{2}$ is the modular parameter of the world-sheet torus, and

$$
\sum_{m, n} Z_{m, n}(\tau, \bar{\tau})=\frac{1}{|\eta(\tau)|^{2}} \sum_{m, n} q^{1 / 2\left(\frac{m}{R}+\frac{n R}{2}\right)^{2}} \bar{q}^{1 / 2\left(\frac{m}{R}-\frac{n R}{2}\right)^{2}}=\frac{R \tau_{2}-1 / 2}{\sqrt{2}|\eta(\tau)|^{2}} \sum_{\tilde{m}, n} e^{-\frac{\pi R^{2}}{2 \tau_{2}}|\tilde{m}+n \tau|^{2}}
$$

where $q=e^{2 i \pi \tau}$. The last form of this expression, obtained by a Poisson resummation in $m$, will be used below to perform the Scherk-Schwarz deformation in a way that keeps modular invariance manifest. On the other hand, the hamiltonian formulation gives the whole expression a lattice interpretation. In this case the partition function takes the form

$$
Z(\tau, \bar{\tau})=\operatorname{Tr} q^{L_{0}} \bar{q}^{\bar{L}_{0}}
$$

where

$$
L_{0}=\frac{1}{2} \mathbf{p}_{L}^{2}+\frac{1}{2}\left(p_{L}^{0}\right)^{2}-\frac{1}{2}+\ldots, \quad \bar{L}_{0}=\frac{1}{2} \mathbf{p}_{R}^{2}+\frac{1}{2}\left(p_{R}^{0}\right)^{2}-\frac{1}{2}+\ldots
$$

\footnotetext{
${ }^{1}$ For brevity, in this Section and in the following we set $\alpha^{\prime}=2$.
} 
Here the dots stand for the contributions of string oscillators, while $p_{L, R}^{0}=m / R \pm n R / 2$ are the left and right momenta associated to the $\Gamma_{(1,1)}$ lattice of the circle. Moreover, we use bosonized fermionic coordinates that give rise to an additional lattice $\Gamma_{(4,4)}$ with momenta $\mathbf{p}_{L, R}$

The general method for breaking supersymmetry by compactification uses a (discrete) R-symmetry of the higher-dimensional theory and couples the lattice momenta to the corresponding R-charges e. The R-transformations are in general discrete remnants of internal rotations. For simplicity, we shall restrict our attention to the $Z_{2}$ case, that corresponds to a $2 \pi$-rotation and therefore affects only spinorial representations. Moreover, this is the only possibility in nine dimensions, where it reduces to fermion number parity. Upon bosonization of the fermionic coordinates, as described above, this construction translates into a Lorentz boost that mixes the $\Gamma_{(1,1)}$ and $\Gamma_{(4,4)}$ lattices consistently with modular invariance:

$$
\begin{array}{cl}
\mathbf{p}_{L} \rightarrow \mathbf{p}_{L}-\boldsymbol{\omega}_{L}\left(p_{L}^{0}-p_{R}^{0}\right), & \mathbf{p}_{R} \rightarrow \mathbf{p}_{R}-\boldsymbol{\omega}_{R}\left(p_{L}^{0}-p_{R}^{0}\right), \\
p_{L}^{0} \rightarrow p_{L}^{0}+\omega \cdot p-\frac{1}{2} \omega \cdot \omega\left(p_{L}^{0}-p_{R}^{0}\right), & p_{R}^{0} \rightarrow p_{R}^{0}+\omega \cdot p-\frac{1}{2} \omega \cdot \omega\left(p_{L}^{0}-p_{R}^{0}\right),
\end{array}
$$

where $\boldsymbol{\omega}=\mathbf{e} / R$ [5]. As usual, the scalar products in (4.5) are defined with a lorentzian metric, so that for instance $\omega \cdot p=\boldsymbol{\omega}_{L} \cdot \mathbf{p}_{L}-\boldsymbol{\omega}_{R} \cdot \mathbf{p}_{R}$. The boost (4.5) translates into the shift:

$$
m \rightarrow m+e \cdot p-\frac{n}{2} e \cdot e, n \rightarrow n, \quad \mathbf{p} \rightarrow \mathbf{p}-n \mathbf{e}
$$

that in the zero winding sector clearly reproduces the standard Scherk-Schwarz momentum shifts of the effective field theory. In this work we are particularly interested in left-right symmetric charge assignments, since these are the starting point for the construction of type-I descendants. In this case, the above equations simplify, since $\mathbf{e}_{L}=\mathbf{e}_{R}$, and therefore $e \cdot e=0$.

The deformed partition function takes a rather compact form in the lagrangian formu- 
lation obtained by the Poisson resummation (4.2):

$$
\begin{aligned}
\mathcal{T}= & \frac{R \tau_{2}^{-4}}{\sqrt{2}|\eta(\tau)|^{24}} \sum_{n, \tilde{m}=-\infty}^{\infty} e^{-\frac{\pi R^{2}}{2 \tau_{2}}|\tilde{m}+n \tau|^{2}} \sum_{\substack{\text { spin } \\
\text { structures }}} \tilde{C}\left(\begin{array}{c}
\mathbf{a}_{L} \\
\mathbf{b}_{L}
\end{array}\right) \tilde{C}^{*}\left(\begin{array}{c}
\mathbf{a}_{R} \\
\mathbf{b}_{R}
\end{array}\right) \times \\
& \prod_{i_{L}=1}^{4} \theta\left(\begin{array}{c}
\mathbf{a}_{i_{L}}-n \mathbf{e}_{i_{L}} \\
\mathbf{b}_{i_{L}}+\tilde{m} \mathbf{e}_{i_{L}}
\end{array}\right)(\tau) \prod_{i_{R}=1}^{4} \bar{\theta}\left(\begin{array}{c}
\mathbf{a}_{i_{R}}-n \mathbf{e}_{i_{R}} \\
\mathbf{b}_{i_{R}}+\tilde{m} \mathbf{e}_{i_{R}}
\end{array}\right)(\bar{\tau}),
\end{aligned}
$$

where

$$
\tilde{C}\left(\begin{array}{c}
\mathbf{a} \\
\mathbf{b}
\end{array}\right)=e^{2 i \pi n \mathbf{e}\left(\mathbf{b}+\frac{\tilde{m}}{2} \mathbf{e}\right)} C\left(\begin{array}{c}
\mathbf{a} \\
\mathbf{b}
\end{array}\right) .
$$

It follows from the partition function, or equivalently from the shifts (4.6), that in nine dimensions this breaking yields mass shifts for all fermions. In particular, the gravitino and all previously massless fermions acquire a mass equal to $e \cdot p / R$.

In the next Section we will extend these results to unoriented closed and open strings ? The standard construction of open descendants [0, 14, 15] then associates to the unoriented sector of the type IIB theory of eq. (4.7) an open sector where supersymmetry is broken by shifts as in eq. (4.6) for all fermions, although with $n=0$. This is effectively the field theory realization of the Scherk-Schwarz mechanism. An even more interesting possibility is realized if the open strings have Dirichlet boundary conditions along the circle. This is the case for type- $\mathrm{I}^{\prime}$ theory, where the circle is identified with the eleventh coordinate of $\mathrm{M}$ theory. For the sake of comparison with the previous, standard case, one can perform a T-duality to revert to the type-I description. In this case the shifts introduced in the closed sector affect the windings, rather than the momenta. Thus, one would expect that the open spectrum be untouched. The explicit analysis of the next Section will show that, while the actual situation is more involved, this naive expectation is realized for the massless modes. The relevant boost for the type IIB theory can be obtained from eq. (4.5) by a T-duality.

\footnotetext{
${ }^{2}$ An alternative way to break supersymmetry using internal magnetic fields is described in [13].
} 
The result is

$$
\begin{aligned}
\mathbf{p}_{L} \rightarrow \mathbf{p}_{L}-\boldsymbol{\eta}_{\boldsymbol{L}}\left(p_{L}^{0}+p_{R}^{0}\right), & \mathbf{p}_{R} \rightarrow \mathbf{p}_{R}-\boldsymbol{\eta}_{\boldsymbol{R}}\left(p_{L}^{0}+p_{R}^{0}\right), \\
p_{L}^{0} \rightarrow p_{L}^{0}+\eta \cdot p-\frac{1}{2} \eta \cdot \eta\left(p_{L}^{0}+p_{R}^{0}\right), & p_{R}^{0} \rightarrow p_{R}^{0}-\eta \cdot p+\frac{1}{2} \eta \cdot \eta\left(p_{L}^{0}+p_{R}^{0}\right),
\end{aligned}
$$

where $\boldsymbol{\eta}=\mathbf{e} R / 2$. The boost (4.9) corresponds to the shift of the lattice momenta

$$
m \rightarrow m, n \rightarrow n+e \cdot p-\frac{m}{2} e \cdot e, \mathbf{p} \rightarrow \mathbf{p}-m \mathbf{e}
$$

clearly related to eq. (4.6) by the interchange of $m$ and $n$. The gravitino mass is now $e \cdot p R / 2$, and supersymmetry is thus recovered in the $R \rightarrow 0$ limit. Finally, the explicit form of the partition function can be obtained from eq. (4.7) replacing $n$ with $m$ and $\tilde{m}$ with $\tilde{n}$, where "tilde" indicates Poisson resummed indices. Again, the type-I descendants are associated to left-right symmetric choices of charges $\mathbf{e}_{L}=\mathbf{e}_{R}$, so that $e \cdot e=0$.

One can actually write the partition function (4.7) and the corresponding one for the shifts (4.10) in a form more suitable for building the open descendants. To this end, let us define the level one $S O(2 n)$ characters

$$
\begin{array}{rlrl}
I_{2 n} & =\frac{1}{2 \eta^{n}}\left(\theta_{3}^{n}+\theta_{4}^{n}\right), & & V_{2 n}=\frac{1}{2 \eta^{n}}\left(\theta_{3}^{n}-\theta_{4}^{n}\right), \\
S_{2 n}=\frac{1}{2 \eta^{n}}\left(\theta_{2}^{n}+i^{n} \theta_{1}^{n}\right), & C_{2 n}=\frac{1}{2 \eta^{n}}\left(\theta_{2}^{n}-i^{n} \theta_{1}^{n}\right),
\end{array}
$$

where $\theta_{i}$ are the four Jacobi theta-functions with integer characteristics. Then, leaving aside all contributions from transverse bosons and from the measure over the moduli, the partition function of the type IIB superstring in 10d is simply

$$
T_{\text {IIB }}=\left|V_{8}-S_{8}\right|^{2}
$$

In this notation, the massless spectrum is manifest; in particular, $\left|V_{8}\right|^{2}$ describes the universal bosonic modes of the NS-NS sector (graviton, dilaton and 2-index antisymmetric tensor), while $\left|S_{8}\right|^{2}$ describes the additional scalar, the 2-form and the self-dual 4-form of the R-R sector. 
In a similar fashion, after a Poisson resummation back to the hamiltonian form, the deformed partition function (4.7) for the ordinary Scherk-Schwarz breaking in nine dimensions with $\mathbf{e}_{L}=\mathbf{e}_{R}=(0,0,0,1)$ becomes

$$
\mathcal{T}_{1}=E_{0}^{\prime}\left(V_{8} \bar{V}_{8}+S_{8} \bar{S}_{8}\right)+O_{0}^{\prime}\left(I_{8} \bar{I}_{8}+C_{8} \bar{C}_{8}\right)-E_{1 / 2}^{\prime}\left(V_{8} \bar{S}_{8}+S_{8} \bar{V}_{8}\right)-O_{1 / 2}^{\prime}\left(I_{8} \bar{C}_{8}+C_{8} \bar{I}_{8}\right)
$$

Here, for brevity, we have introduced the projected lattice sums

$$
\begin{array}{ll}
E_{0}^{\prime}=\sum_{m, n} \frac{1+(-1)^{n}}{2} Z_{m, n}, & O_{0}^{\prime}=\sum_{m, n} \frac{1-(-1)^{n}}{2} Z_{m, n}, \\
E_{1 / 2}^{\prime}=\sum_{m, n} \frac{1+(-1)^{n}}{2} Z_{m+1 / 2, n}, & O_{1 / 2}^{\prime}=\sum_{m, n} \frac{1-(-1)^{n}}{2} Z_{m+1 / 2, n},
\end{array}
$$

where $E^{\prime}$ and $O^{\prime}$ refer to even and odd windings and the subscripts 0 and $1 / 2$ refer to unshifted and shifted momenta. In this string generalization of the Scherk-Schwarz mechanism, all space-time fermions have evidently masses shifted by $1 /(2 R)$ compared to the supersymmetric case. Modular invariance, however, changes the GSO projection in the odd winding sector. As a result, for $R \leq \sqrt{\alpha^{\prime}}$ the spectrum contains a tachyon associated to $I_{8} \bar{I}_{8}$. [ The supersymmetric type IIB model (4.12) in $10 \mathrm{~d}$ is recovered in the $R \rightarrow \infty$ limit.

Alternatively, this model can be obtained as an asymmetric orbifold of the non-supersymmetric 0B model with partition function

$$
T_{0 \mathrm{~B}}=\left|I_{8}\right|^{2}+\left|V_{8}\right|^{2}+\left|S_{8}\right|^{2}+\left|C_{8}\right|^{2}
$$

using the discrete symmetry $g=-(-1)^{G_{L}}(-1)^{n}$, where $G_{L}$ is the world-sheet left fermion number, so that $-(-1)^{G_{L}}$ acts as 1 on $V_{8}, S_{8}$ and -1 on $I_{8}, C_{8}$. This model can also be described as a symmetric orbifold of the IIB superstring of eq. (4.12) by the $Z_{2}$ symmetry $(-1)^{F} I$, where $F=F_{L}+F_{R}$ is the space-time fermion number and the shift $I: X_{9} \rightarrow$ $X_{9}+\pi R$ acts on states as $(-1)^{m}$. The resulting partition function $\mathcal{T}_{1}^{\prime}$ coincides with $\mathcal{T}_{1}$ upon doubling the radius, so that $\mathcal{T}_{1}(R)=\mathcal{T}_{1}^{\prime}(2 R)$. More explicitly

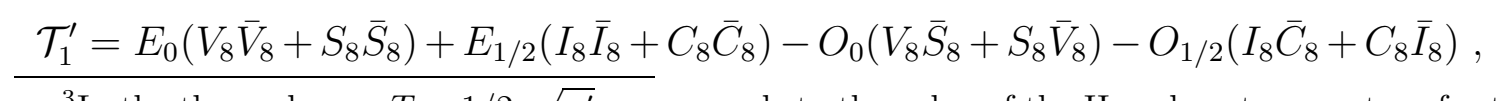

${ }^{3}$ In the thermal case, $T=1 / 2 \pi \sqrt{\alpha^{\prime}}$ corresponds to the value of the Hagedorn temperature for the type II theory. 
where $E_{0}, O_{0}, E_{1 / 2}, O_{1 / 2}$ are defined as in eq. (4.14) but with $m$ and $n$ interchanged.

Finally, the type IIB deformed model with windings shifted according to eq. (4.10) can be obtained in a straightforward way from eq. (4.13) interchanging momenta and windings. The resulting partition function is

$$
\mathcal{T}_{2}=E_{0}\left(V_{8} \bar{V}_{8}+S_{8} \bar{S}_{8}\right)+O_{0}\left(I_{8} \bar{I}_{8}+C_{8} \bar{C}_{8}\right)-E_{1 / 2}\left(V_{8} \bar{S}_{8}+S_{8} \bar{V}_{8}\right)-O_{1 / 2}\left(I_{8} \bar{C}_{8}+C_{8} \bar{I}_{8}\right)
$$

This model is tachyon-free for values of the radius $R \leq \sqrt{\alpha^{\prime}}$, while the supersymmetric 10d IIB theory is recovered in the $R \rightarrow 0$ limit. A field theory interpretation is thus more natural in the T-dual type-IIA description, where the shifts are transferred back to the momenta. The corresponding open sector, however, corresponds to the type I' construction with Dirichlet boundary conditions. Since the resulting open strings have only windings, the shifted momenta are naturally associated to the eleventh direction of $\mathrm{M}$ theory, as we have shown in Section 3. In the following, we shall refer to this model as the M-theory breaking model. Note that it can also be obtained as an asymmetric orbifold of the 0B theory (4.15), using the discrete symmetry $g=-(-1)^{G_{L}} I$.

\section{Explicit type I models in nine dimensions}

\subsection{Review of the construction procedure}

Before describing the nine-dimensional type I models, we would like to present a brief review of the algorithm that we use. This was introduced in [14, 15], and developed further in [16]. Type I models can be obtained as "orbifolds" of left-right symmetric type-IIB models by the world-sheet involution $\Omega$ that interchanges left and right movers [7]. The starting point

thus consists in adding to the (halved) torus amplitude the Klein-bottle $\mathcal{K}$. This completes the projection induced by $\Omega$, and is a linear combination of the diagonal contributions to 
the torus amplitude, with argument $q \bar{q}$, where $q$ was defined after eq. (4.1). Thus, starting from

$$
\mathcal{T}=\sum_{i j} X_{i j} \chi_{i}(\tau) \chi_{j}(\bar{\tau})
$$

where the $\chi$ 's are a set of characters of the underlying conformal field theory and $X$ is a matrix of integers, one obtains f

$$
\mathcal{K}=\frac{1}{2} \sum_{i} X_{i i} \chi_{i}\left(2 i \tau_{2}\right)
$$

with $\tau_{2}$ the proper time for the closed string. In order to identify the corresponding open sector, it is useful to perform the $S$ modular transformation induced by

$$
\mathcal{K}: \quad 2 \tau_{2} \underset{s}{\longrightarrow} \frac{1}{2 \tau_{2}} \equiv l
$$

thus turning the direct-channel Klein-bottle amplitude $\mathcal{K}$ into the transverse-channel amplitude $\tilde{\mathcal{K}}$. The latter describes the propagation of the closed spectrum on a tube of length $l$ terminating at two crosscaps?, and has the generic form

$$
\tilde{\mathcal{K}}=\sum_{i} \Gamma_{i}^{2} \chi_{i}(i l)
$$

where the coefficients $\Gamma_{i}$ can be related to the one-point functions of the closed-string fields of $\mathcal{T}$ in the presence of a crosscap.

The open strings correspond to the twisted sector of the spectrum with respect to the involution $\Omega$, and may be deduced from the closed-string spectrum in a similar fashion. First, the direct-channel annulus amplitude $\mathcal{A}$ may be deduced from the transverse-channel boundary-to-boundary amplitude $\tilde{\mathcal{A}}$. This has the general form [15

$$
\tilde{\mathcal{A}}=\sum_{i} B_{i}^{2} \chi_{i}(i l)
$$

\footnotetext{
${ }^{4}$ As discussed in [16], in general one has the option to modify eq. (5.2), altering $X_{i i}$ by signs $\epsilon_{i}$. These turn sectors symmetrized under left-right interchange into antisymmetrized ones, and vice-versa, and are in general constrained by compatibility with the fusion rules.

${ }^{5}$ The crosscap, or real projective plane, is a non-orientable surface that may be defined starting from a 2-sphere and identifying antipodal points.
} 
where the coefficients $B_{i}$ can be related to the one-point functions of closed-string fields in the presence of boundaries. The relevant $S$ modular transformation now maps the closed string proper time $l$ on the tube into the open-string proper time $t$ on the annulus according to

$$
\mathcal{A}: \quad l \underset{s}{\rightarrow} \frac{1}{l} \equiv \frac{t_{\mathcal{A}}}{2}
$$

The direct-channel annulus amplitude then takes the form

$$
\mathcal{A}=\frac{1}{2} \sum_{i, a, b} A_{a b}^{i} n_{a} n_{b} \chi_{i}\left(\frac{i t_{\mathcal{A}}}{2}\right),
$$

where the $n$ 's are integers that have the interpretation of Chan-Paton multiplicities for the boundaries and the $A^{i}$ are a set of matrices with integer elements. These matrices are obtained solving diophantine equations determined by the condition that the modular transform of eq. (5.5) involve only integer coefficients, while the Chan-Paton multiplicities arise as free parameters of the solution.

Finally, the transverse-channel Möbius amplitude $\tilde{\mathcal{M}}$ describes the propagation of closed strings between a boundary and a crosscap, and is determined by factorization from $\tilde{\mathcal{K}}$ and $\tilde{\mathcal{A}}$. It contains the characters common to the two expressions with coefficients that are geometric means of those present in $\tilde{\mathcal{K}}$ and $\tilde{\mathcal{A}}[15]$. Thus

$$
\tilde{\mathcal{M}}=2 \sum_{i} B_{i} \Gamma_{i} \hat{\chi}_{i}\left(i l+\frac{1}{2}\right),
$$

where the hatted characters form a real basis and are obtained by the redefinitions

$$
\hat{\chi}_{i}\left(i l+\frac{1}{2}\right)=e^{-i \pi h_{i}} \chi_{i}\left(i l+\frac{1}{2}\right)
$$

where $h_{i}$ are the conformal weights of the corresponding primary fields. The direct-channel Möbius amplitude $\mathcal{M}$ can then be related to $\tilde{\mathcal{M}}$ by a modular $P$ transformation and a redefinition

$$
\mathcal{M}: \quad \frac{i t_{\mathcal{M}}}{2}+1 / 2 \underset{P}{\longrightarrow} \frac{i}{2 t_{\mathcal{M}}}+\frac{1}{2} \equiv i l+\frac{1}{2}
$$


This is realized on the hatted characters by the sequence $P=T^{1 / 2} S T^{2} S T^{1 / 2}$, with $T$ the diagonal matrix that implements the transformation $\tau \rightarrow \tau+1$. The direct-channel Möbius amplitude then takes the form

$$
\mathcal{M}=\frac{1}{2} \sum_{i, a} M_{a}^{i} n_{a} \hat{\chi}_{i}\left(\frac{i t_{\mathcal{M}}}{2}+\frac{1}{2}\right)
$$

where by consistency the integer coefficients $M_{a}^{i}$ satisfy the constraints

$$
M_{a}^{i}=A_{a a}^{i} \quad(\bmod 2)
$$

that make $\mathcal{M}$ the completion of $\mathcal{A}$. The full one-loop vacuum amplitude is then

$$
\int\left(\frac{1}{2} \mathcal{T}(\tau, \bar{\tau})+\mathcal{K}\left(2 i \tau_{2}\right)+\mathcal{A}\left(\frac{i t}{2}\right)+\mathcal{M}\left(\frac{i t}{2}+\frac{1}{2}\right)\right)
$$

where the different measures of integration are left implicit. In the remainder of this paper, we shall often omit the dependence on world-sheet modular parameters.

For the models of interest, in order to link the direct and transverse channels, one needs the transformation matrices $S$ and $P$ for the level-one $S O(2 n)$ characters of eq. (4.11). These may be simply deduced from the corresponding transformation properties of the Jacobi theta functions, and are

$$
S_{(2 n)}=\frac{1}{2}\left(\begin{array}{cccc}
1 & 1 & 1 & 1 \\
1 & 1 & -1 & -1 \\
1 & -1 & i^{-n} & -i^{-n} \\
1 & -1 & -i^{-n} & i^{-n}
\end{array}\right), P_{(2 n)}=\left(\begin{array}{cccc}
c & s & 0 & 0 \\
s & -c & 0 & 0 \\
0 & 0 & \zeta c & i \zeta s \\
0 & 0 & i \zeta s & \zeta c
\end{array}\right),
$$

where $c=\cos n \pi / 4, s=\sin n \pi / 4$ and $\zeta=e^{-i n \pi / 4}$.

For later use, let us also define

$$
Z_{m+a}(\tau)=\frac{q^{\frac{1}{2}\left(\frac{m+a}{R}\right)^{2}}}{\eta(\tau)}, \quad \tilde{Z}_{n+b}(\tau)=\frac{q^{\frac{1}{2}\left((n+b) \frac{R}{2}\right)^{2}}}{\eta(\tau)}
$$

In relating the direct and transverse channels, one also needs the Poisson transformation

$$
\sum_{m} e^{2 i \pi m b} Z_{m+a}\left(-\frac{1}{\tau}\right)=R e^{-2 i \pi a b} \sum_{n} e^{-2 i \pi n a} \tilde{Z}_{2 n+2 b}(\tau)
$$




\subsection{Scherk-Schwarz breaking model}

We can now proceed to construct the open descendants of the two deformed type IIB models described by $\mathcal{T}_{1}$ and $\mathcal{T}_{2}$ of eqs. (4.13) and (4.17). To this end, we begin by applying the $\Omega$ projection, that symmetrizes the NS-NS sector and antisymmetrizes the R-R sector. The Klein bottle contribution to the partition function completes the projection of the closed sector, and thus receives contributions from all sectors invariant under $\Omega$. These include, in particular, the sublattice

$$
\mathbf{p}_{L}=\mathbf{p}_{R}, p_{L}^{0}=p_{R}^{0}
$$

It is then clear that for the Scherk-Schwarz breaking model (4.13) the Klein bottle is unaffected by supersymmetry breaking. Indeed, the shifts of eq. (4.6) vanish identically, since $e \cdot p=e \cdot e=0$ for the states satisfying the conditions (5.17). The resulting Klein bottle projection $\mathcal{K}_{1}$ is

$$
\mathcal{K}_{1}=\frac{1}{2}\left(V_{8}-S_{8}\right) \sum_{m} Z_{m}
$$

where we have left implicit an overall factor $\tau_{2}^{-11 / 2} \eta^{-7} d \tau_{2}$. The transverse-channel Klein bottle amplitude is then

$$
\tilde{\mathcal{K}}_{1}=\frac{2^{9 / 2}}{2}\left(V_{8}-S_{8}\right) R \sum_{n} \tilde{Z}_{2 n}
$$

where the numerical factor $2^{9 / 2}$ originates from the relation (5.3) between $\tau_{2}$ and $l$, after taking into account all implicit factors in the measure over the moduli.

The transverse-channel annulus amplitude is determined by $\mathcal{T}_{1}$ of eq. (4.13) restricting the diagonal portion of the spectrum to the zero-momentum $(m=0)$ sector. Thus, only $V_{8}$

and $S_{8}$ with even windings and $I_{8}$ and $C_{8}$ with odd ones are allowed in $\tilde{\mathcal{A}}$. We then associate to these terms the minimal number of independent reflection coefficients, parametrized in terms of four integers $\left(n_{1}, n_{2}, n_{3}, n_{4}\right)$, and write

$$
\begin{aligned}
\tilde{\mathcal{A}}_{1} & =\frac{2^{-11 / 2} R}{2}\left(\left[\left(n_{1}+n_{2}+n_{3}+n_{4}\right)^{2} V_{8}-\left(n_{1}+n_{2}-n_{3}-n_{4}\right)^{2} S_{8}\right] \tilde{Z}_{2 n}\right. \\
& \left.+\left[\left(n_{1}-n_{2}+n_{3}-n_{4}\right)^{2} I_{8}-\left(n_{1}-n_{2}-n_{3}+n_{4}\right)^{2} C_{8}\right] \tilde{Z}_{2 n+1}\right),
\end{aligned}
$$


where from now on, for the sake of brevity, we shall often leave lattice sums implicit. This parametrization solves the constraints of eq. (5.7) and leads, via the $S_{(8)}$ transformation (5.14), to the direct-channel annulus amplitude

$$
\begin{aligned}
\mathcal{A}_{1} & =\frac{n_{1}^{2}+n_{2}^{2}+n_{3}^{2}+n_{4}^{2}}{2}\left(V_{8} Z_{2 m}-S_{8} Z_{2(m+1 / 2)}\right)+\left(n_{1} n_{2}+n_{3} n_{4}\right)\left(V_{8} Z_{2(m+1 / 2)}-S_{8} Z_{2 m}\right)+ \\
& +\left(n_{1} n_{3}+n_{2} n_{4}\right)\left(I_{8} Z_{2 m}-C_{8} Z_{2(m+1 / 2)}\right)+\left(n_{1} n_{4}+n_{2} n_{3}\right)\left(I_{8} Z_{2(m+1 / 2)}-V_{8} Z_{2 m}\right),(5.21)
\end{aligned}
$$

that describes an open spectrum with four types of Chan-Paton charges. As we shall see below, additional reflection coefficients would correspond to the introduction of Wilson lines.

Finally, the characters common to $\tilde{\mathcal{K}}_{1}$ and $\tilde{\mathcal{A}}_{1}$ determine the transverse Möbius amplitude

$$
\begin{aligned}
\tilde{\mathcal{M}}_{1}= & -\frac{R}{\sqrt{2}}\left(\left(n_{1}+n_{2}+n_{3}+n_{4}\right) \hat{V}_{8}\left(\tilde{Z}_{4 n}+\tilde{Z}_{4 n+2}\right)\right. \\
& \left.-\left(n_{1}+n_{2}-n_{3}-n_{4}\right) \hat{S}_{8}\left(\tilde{Z}_{4 n}-\tilde{Z}_{4 n+2}\right)\right)
\end{aligned}
$$

where, as in all subsequent Möbius amplitudes, $\tilde{Z}_{n}$ (or $Z_{m}$ ) are actually "hatted" Virasoro characters. The relative signs between the terms proportional to $\tilde{Z}_{4 n}$ and to $\tilde{Z}_{4 n+2}$, left undetermined by the geometric mean leading to $\tilde{\mathcal{M}}$, are fixed by the constraint (5.12). Indeed, the naive choice of all equal signs would lead to a Möbius amplitude incompatible with the particle interpretation of the open sector. This is often the case in these constructions: eq. (5.12) fixes the vacuum channel Möbius amplitude $\tilde{\mathcal{M}}$ that, in contrast to $\tilde{\mathcal{K}}$ and $\tilde{\mathcal{A}}$, is not restricted by positivity. The direct-channel Möbius amplitude is then obtained by the $P_{(8)}$ transformation (5.14), and is given by

$$
\mathcal{M}_{1}=-\frac{n_{1}+n_{2}+n_{3}+n_{4}}{2} \hat{V}_{8} Z_{2 m}+\frac{n_{1}+n_{2}-n_{3}-n_{4}}{2} \hat{S}_{8} Z_{2(m+1 / 2)} .
$$

From eqs. (5.21) and (5.23), we see that the direct-channel Möbius contribution is the proper (anti)symmetrization of the terms in the annulus amplitude describing open strings with pairs of identical charges at their ends. Note that $\mathcal{M}_{1}$ contains an additional factor 
1/2 compared to $\tilde{\mathcal{M}}_{1}$. This rescaling is not a consequence of the $P$-transformation, but is induced by the redefinition of the functional measure in going from the transverse-channel variable $l \rightarrow l / 2$.

The tadpole conditions are obtained from $\tilde{K}_{1}, \tilde{A}_{1}$ and $\tilde{M}_{1}$, setting to zero the total reflection coefficients for the massless modes, that for generic radii originate from $V_{8}$ and $S_{8}$, and read:

$$
\begin{array}{ll}
V_{8}: & \frac{2^{9 / 2}}{2}+\frac{2^{-11 / 2}}{2}\left(n_{1}+n_{2}+n_{3}+n_{4}\right)^{2}-\frac{1}{\sqrt{2}}\left(n_{1}+n_{2}+n_{3}+n_{4}\right)=0 \\
S_{8}: & \frac{2^{9 / 2}}{2}+\frac{2^{-11 / 2}}{2}\left(n_{1}+n_{2}-n_{3}-n_{4}\right)^{2}-\frac{1}{\sqrt{2}}\left(n_{1}+n_{2}-n_{3}-n_{4}\right)=0
\end{array}
$$

so that

$$
n_{1}+n_{2}+n_{3}+n_{4}=32, \quad n_{1}+n_{2}-n_{3}-n_{4}=32
$$

Thus, $n_{3}=n_{4}=0$ and $n_{1}+n_{2}=32$. It should be appreciated that all tachyons originating from the $I_{8} Z_{2 m}$ sector are then removed. The resulting open spectrum

$$
\begin{aligned}
\mathcal{A}_{1} & =\frac{n_{1}^{2}+n_{2}^{2}}{2}\left(V_{8} Z_{2 m}-S_{8} Z_{2(m+1 / 2)}\right)+n_{1} n_{2}\left(V_{8} Z_{2(m+1 / 2)}-S_{8} Z_{2 m}\right), \\
\mathcal{M}_{1} & =-\frac{n_{1}+n_{2}}{2}\left(\hat{V}_{8} Z_{2 m}-\hat{S}_{8} Z_{2(m+1 / 2)}\right),
\end{aligned}
$$

corresponds to a family of gauge groups $S O\left(n_{1}\right) \times S O\left(n_{2}\right)$, with $n_{1}+n_{2}=32$. For interger momentum levels f the spectrum consists of vectors $\overline{7}$ in the representations $\left(\mathbf{n}_{\mathbf{1}}\left(\mathbf{n}_{\mathbf{1}}-\mathbf{1}\right) / \mathbf{2}, \mathbf{1}\right)$ $+\left(\mathbf{1}, \mathbf{n}_{\mathbf{2}}\left(\mathbf{n}_{\mathbf{2}}-\mathbf{1}\right) / \mathbf{2}\right)$ and fermions in the representation $\left(\mathbf{n}_{\mathbf{1}}, \mathbf{n}_{\mathbf{2}}\right)$. On the other hand, for halfinteger levels, the spectrum consists of fermions in the $\left(\mathbf{n}_{\mathbf{1}}\left(\mathbf{n}_{\mathbf{1}}-\mathbf{1}\right) / \mathbf{2}, \mathbf{1}\right)+\left(\mathbf{1}, \mathbf{n}_{\mathbf{2}}\left(\mathbf{n}_{\mathbf{2}}-\mathbf{1}\right) / \mathbf{2}\right)$ and vectors in the $\left(\mathbf{n}_{\mathbf{1}}, \mathbf{n}_{\mathbf{2}}\right)$. This partial breaking results from Wilson lines [17] in the original $S O(32)$ gauge group, a subject to which we now turn.

\footnotetext{
${ }^{6}$ According to our definitions $(5.6),(5.10)$ and eq. (5.15), in the open spectrum $Z_{2 m}$ describes states of integer momenta with masses $m / R$, while $Z_{2(m+1 / 2)}$ describes states of half-integer momenta.

${ }^{7}$ In $9 \mathrm{~d}$ the vector $V_{8}$ is a reducible representation, and already at the lowest level it describes a vector $A_{\mu}$ and a scalar $A_{9}$.
} 
Our aim is now to compare this class of models with another obtained from the type-I $S O(32)$ model breaking in part the gauge symmetry without affecting the supersymmetry since, as we have anticipated, the model (5.26) originates from a discrete deformation that induces the spontaneous breaking of supersymmetry. It is thus useful to recall briefly how Wilson lines can be introduced in open strings [17], considering the simplest example of the circle compactification of the type I superstring to $9 \mathrm{~d}$. A Wilson line $W$ originating from a gauge field background $a=\operatorname{diag}\left(a_{1},-a_{1} \cdots a_{16},-a_{16}\right)=\left(a_{i}\right)_{i=1 \cdots 32}$ in the Cartan subalgebra of $S O(32), W=\operatorname{diag}\left(e^{2 \pi i a_{1}}, e^{-2 \pi i a_{1}} \cdots e^{2 \pi i a_{16}}, e^{-2 \pi i a_{16}}\right)$, generically breaks $S O(32)$ to $U(1)^{16}$, while special values of the parameters can break $S O(32)$ into products of even orthogonal subgroups ${ }^{8}$. The resulting model is

$$
\begin{array}{ll}
\mathcal{T}=\left|V_{8}-S_{8}\right|^{2} Z_{m, n}, & \mathcal{K}=\frac{1}{2}\left(V_{8}-S_{8}\right) Z_{m} \\
\mathcal{A}=\frac{1}{2}\left(V_{8}-S_{8}\right) \sum_{i, j=1}^{32} Z_{2\left(m+a_{i}+a_{j}\right)}, & \mathcal{M}=-\frac{1}{2}\left(\hat{V}_{8}-\hat{S}_{8}\right) \sum_{i=1}^{32} Z_{2\left(m+2 a_{i}\right)}
\end{array}
$$

In the transverse channel the amplitudes take the elegant form

$$
\begin{aligned}
\tilde{\mathcal{K}} & =\frac{2^{9 / 2}}{2} R\left(V_{8}-S_{8}\right) \tilde{Z}_{2 n}, \\
\tilde{\mathcal{A}} & =\frac{2^{-11 / 2}}{2} R\left(V_{8}-S_{8}\right) \sum_{n}\left(\operatorname{Tr} W^{n}\right)^{2} \tilde{Z}_{n}, \\
\tilde{\mathcal{M}} & =-\frac{R}{\sqrt{2}}\left(\hat{V}_{8}-\hat{S}_{8}\right) \sum_{n} \operatorname{Tr} W^{2 n} \tilde{Z}_{2 n},
\end{aligned}
$$

where, for instance

$$
\sum_{i, j} e^{2 \pi i n\left(a_{i}+a_{j}\right)}=\left(\operatorname{Tr} W^{n}\right)^{2}
$$

We can thus break the gauge group to $S O\left(n_{1}\right) \times S O\left(32-n_{1}\right)$ with $n_{1}$ even (or odd with a parity-like $W$ ) [17], and the resulting amplitudes

$$
\mathcal{K}=\frac{1}{2}\left(V_{8}-S_{8}\right) Z_{m}
$$

\footnotetext{
${ }^{8}$ Odd orthogonal subgroups can also be obtained, by discrete deformations with matrices $W$ in $O(32)$, with determinant equal to -1 .
} 


$$
\begin{aligned}
\mathcal{A} & =\left(V_{8}-S_{8}\right)\left(\frac{n_{1}^{2}+n_{2}^{2}}{2} Z_{2 m}+n_{1} n_{2} Z_{2(m+1 / 2)}\right), \\
\mathcal{M} & =-\frac{n_{1}+n_{2}}{2}\left(V_{8}-S_{8}\right) Z_{2 m},
\end{aligned}
$$

show clearly that the model $(\underline{5.26})$ is a discrete deformation (resulting in a discrete mass shift, $m \rightarrow m+1 / 2$, for the fermion spectrum) of the supersymmetric model (5.30). This reflects the spontaneous character of the breaking, that disappears in the limit $R \rightarrow \infty$. In retrospect, this result is a natural extension of the boost 4.5) responsible for the ScherkSchwarz breaking in the type-IIB string to the open sector. Indeed, in the closed sector the boost may be seen as a left-right symmetric discrete Wilson line built from the graviphoton. The discrete shifts (4.6) in the closed fermion spectrum have thus counterparts in the open sector. Note that this model describes also type I theory at finite temperature, upon the identification of the compact coordinate with euclidean time, $T=1 /(2 \pi R)$.

One can also introduce continuous Wilson lines starting from the model (5.26) with $n_{2}=0$ to induce various breakings of the gauge group. For instance, 16 pairs of different parameters $\left(a_{i},-a_{i}\right)$ lead to

$$
\begin{aligned}
\mathcal{A}_{1} & =\frac{1}{2} \sum_{i, j=1}^{32}\left(V_{8} Z_{2\left(m+a_{i}+a_{j}\right)}-S_{8} Z_{2\left(m+1 / 2+a_{i}+a_{j}\right)}\right) \\
\mathcal{M}_{1} & =-\frac{1}{2} \sum_{i=1}^{32}\left(V_{8} Z_{2\left(m+2 a_{i}\right)}-S_{8} Z_{2\left(m+1 / 2+2 a_{i}\right)}\right)
\end{aligned}
$$

and the $S O(32)$ gauge group is correspondingly broken to $U(1)^{16}$. The models (5.26) with even $n_{2}$ can be recovered as special cases if $n_{2} / 2$ of the $a_{i}$ equal $1 / 2, n_{2} / 2$ equal $-1 / 2$ and the rest vanish. Alternatively, for arbitrary $a_{i}$ this model corresponds to a Scherk-Schwarz discrete deformation of (5.27), whereby the momenta of the fermionic modes are shifted by 1/2 unit. As mentioned in Section 3, this class of models is dual to corresponding ScherkSchwarz compactifications of the $S O(32)$ heterotic string, whose gauge sector coincides with (5.31) when restricted to zero windings.

For later use, we now show a simple instance of gauge symmetry breaking to unitary 
groups, and thus a spectrum with oriented open strings. Starting again from $n_{2}=0$, this

corresponds to $W=\operatorname{diag}\left(e^{2 \pi i / 4}, e^{-2 \pi i / 4} \cdots e^{2 \pi i / 4}, e^{-2 \pi i / 4}\right)$, that breaks $S O(32)$ to $U(16)$.

The resulting open sector is

$$
\begin{aligned}
\mathcal{A}_{1} & =n \bar{n}\left(V_{8} Z_{2 m}-S_{8} Z_{2(m+1 / 2)}\right)+\frac{n^{2}+\bar{n}^{2}}{2}\left(V_{8} Z_{2(m+1 / 2)}-S_{8} Z_{2 m}\right) \\
\mathcal{M}_{1} & =-\frac{n+\bar{n}}{2}\left(V_{8} Z_{2(m+1 / 2)}-S_{8} Z_{2 m}\right)
\end{aligned}
$$

Here $n(=\bar{n})$ label a pair of "complex" charges, corresponding to the fundamental representation of $U(n)$ and its conjugate [15]. The tadpole conditions then fix $n=16$. Again, there are no open-string tachyons. For integer momenta, the spectrum consists of a vector in the adjoint and of a spinor in the antisymmetric $\mathbf{n}(\mathbf{n}-\mathbf{1}) / \mathbf{2}$ and its conjugate. For half-integer momenta, the spectrum consists of a vector in the antisymmetric $\mathbf{n}(\mathbf{n}-\mathbf{1}) / \mathbf{2}$ and its conjugate, and of a spinor in the adjoint. This model, as well as special cases of eq. (5.26), were obtained previously (with double radius) in 18, starting from the orbifold $\mathcal{T}_{1}^{\prime}$ of the IIB superstring in eq. (4.16).

\subsection{M-theory breaking model}

Starting from the torus amplitude $\mathcal{T}_{2}$ of eq. (4.17) with shifts on the windings and following the same steps as before, one obtains

$$
\mathcal{K}_{2}=\frac{1}{2}\left(V_{8}-S_{8}\right) Z_{2 m}+\frac{1}{2}\left(I_{8}-C_{8}\right) Z_{2 m+1}
$$

and consequently

$$
\tilde{\mathcal{K}}_{2}=\frac{2^{9 / 2}}{2} R\left(V_{8} \tilde{Z}_{2 n}-S_{8} \tilde{Z}_{2 n+1}\right)
$$

In contrast to the previous case, now the Klein bottle is affected by the deformation.

The transverse annulus amplitude is determined from $\mathcal{T}_{2}$ of eq. (4.17), restricting the diagonal portion of the spectrum to zero momentum $(m=0)$. There are thus four independent reflection coefficients associated to $V_{8}$ and $S_{8}$ with even and with odd windings. 
As before, they can be parametrized in terms of four integers $\left(n_{1}, n_{2}, n_{3}, n_{4}\right)$, so that

$$
\begin{aligned}
\tilde{\mathcal{A}}_{2} & =\frac{2^{-11 / 2}}{2} R\left(\left[\left(n_{1}+n_{2}+n_{3}+n_{4}\right)^{2} V_{8}-\left(n_{1}+n_{2}-n_{3}-n_{4}\right)^{2} S_{8}\right] \tilde{Z}_{2 n}\right. \\
& \left.+\left[\left(n_{1}-n_{2}+n_{3}-n_{4}\right)^{2} V_{8}-\left(n_{1}-n_{2}-n_{3}+n_{4}\right)^{2} S_{8}\right] \tilde{Z}_{2 n+1}\right),
\end{aligned}
$$

and the corresponding direct-channel annulus amplitude is

$$
\begin{aligned}
\mathcal{A}_{2} & =\left(\frac{n_{1}^{2}+n_{2}^{2}+n_{3}^{2}+n_{4}^{2}}{2}\left(V_{8}-S_{8}\right)+\left(n_{1} n_{3}+n_{2} n_{4}\right)\left(I_{8}-C_{8}\right)\right) Z_{2 m} \\
& +\left(\left(n_{1} n_{2}+n_{3} n_{4}\right)\left(V_{8}-S_{8}\right)+\left(n_{1} n_{4}+n_{2} n_{3}\right)\left(I_{8}-C_{8}\right)\right) Z_{2(m+1 / 2)}
\end{aligned}
$$

The characters common to $\tilde{K}_{2}$ and $\tilde{A}_{2}$ then determine the transverse-channel Möbius amplitude

$$
\begin{aligned}
& \tilde{\mathcal{M}}_{2}=-\frac{R}{\sqrt{2}}\left(\left(n_{1}+n_{2}+n_{3}+n_{4}\right) \hat{V}_{8} \tilde{Z}_{2 n}-\left(n_{1}-n_{2}-n_{3}+n_{4}\right) \hat{S}_{8} \tilde{Z}_{2 n+1}\right) \\
& \mathcal{M}_{2}=-\frac{n_{1}+n_{2}+n_{3}+n_{4}}{2} \hat{V}_{8} Z_{2 m}+\frac{n_{1}-n_{2}-n_{3}+n_{4}}{2} \hat{S}_{8}(-1)^{m} Z_{2 m}
\end{aligned}
$$

where the implicit summation over $m$ now contains alternating signs in the last term.

The tadpole conditions, obtained setting to zero the coefficients of the massless modes originating from $V_{8}$ and $S_{8}$, give

$$
n_{1}+n_{2}+n_{3}+n_{4}=32, \quad n_{1}+n_{2}-n_{3}-n_{4}=0
$$

Here, however, one needs further conditions to remove the tachyon from the $I_{8} Z_{m}$ sector in $\mathcal{A}$, and a particularly interesting solution corresponds to $n_{2}=n_{3}=0$. The result is the unique gauge group $S O(16) \times S O(16)$, and the open sector reads

$$
\begin{aligned}
\mathcal{A}_{2} & =\frac{n_{1}^{2}+n_{4}^{2}}{2}\left(V_{8}-S_{8}\right) Z_{2 m}+n_{1} n_{4}\left(I_{8}-C_{8}\right) Z_{2(m+1 / 2)}, \\
\mathcal{M}_{2} & =-\frac{n_{1}+n_{4}}{2} \hat{V}_{8} Z_{2 m}+\frac{n_{1}+n_{4}}{2} \hat{S}_{8}(-1)^{m} Z_{2 m} .
\end{aligned}
$$

Notice that the massless open spectrum is actually supersymmetric, since

$$
\begin{aligned}
\mathcal{A}_{2} & =\frac{n_{1}^{2}+n_{4}^{2}}{2}\left(V_{8}-S_{8}\right)+\text { massive } \\
\mathcal{M}_{2} & =-\frac{n_{1}+n_{4}}{2}\left(\hat{V}_{8}-\hat{S}_{8}\right)+\text { massive } .
\end{aligned}
$$


Actually, the open spectrum is supersymmetric for even momenta, and in terms of $S O(8)$ representations describes a vector and a spinor in the adjoint of $S O(16) \times S O(16)$. On the other hand, for odd momenta the vector is again in the adjoint, while the spinor is in the symmetric representations $(\mathbf{1 3 5}, \mathbf{1})+2(\mathbf{1}, \mathbf{1})+(\mathbf{1}, \mathbf{1 3 5})$. Finally, for half-integer momenta, there are scalars and spinors in the $(\mathbf{1 6}, \mathbf{1 6})$ representation.

The duality arguments of Section 3 associate the closed sector of this type-I model, after a T-duality, to a Scherk-Schwarz deformation affecting the momenta of the type-IIA string. In the corresponding type-I' representation, however, the open strings end on D8-branes perpendicular to the direction responsible for the breaking of supersymmetry. Therefore, as we have just seen, all open string modes with even windings, and in particular the massless ones, are unaffected. On the other hand, both the changes in the open spectrum for the states with odd windings and the presence of half-integer windings can be traced to corresponding modifications of the closed spectrum due to the reversal of the GSO projection. This can be seen from eq. (4.10), after interchanging $m$ and $n$. The soft nature of this breaking is less evident than in the previous example. In the next Section, however, we will show that the one-loop contribution of the open string sector to the vacuum energy and to the scalar masses is exponentially suppressed in the decompactification limit, that in type I language corresponds to $R \rightarrow 0$. Resorting again to the duality arguments of Section 3 , it is clear that this breaking corresponds to a non-perturbative phenomenon on the heterotic side and realizes the Scherk-Schwarz deformation along the eleventh coordinate of $\mathrm{M}$ theory.

The tadpole equations (5.38) admit a second tachyon-free solution, $n_{1}=n_{4}=0$ and $n_{2}=n_{3}=16$. It is then easy to see that the open-string sector is identical to that of eq. (5.39), with $n_{2}$ and $n_{3}$ playing the role of $n_{1}$ and $n_{4}$, aside from a crucial relative sign change between the $\hat{V}_{8}$ and $\hat{S}_{8}$ contributions to $\mathcal{M}_{2}$. The resulting gauge group is again $S O(16) \times S O(16)$, but now supersymmetry is broken already at the massless level, since the 
fermion representations corresponding to even and odd values of momenta are interchanged. While this model is perturbatively consistent, it is not clear to us what its dual M-theory interpretation is. Moreover, if one requires, in the spirit of [9], that all tadpoles that become massless in the $R \rightarrow 0$ limit vanish, one is left with the unique choice $n_{1}=n_{4}=16$ and $n_{2}=n_{3}=0$, and the end result is the M-theory breaking model. Furthermore, there are other choices for the Klein-bottle projection for both models, that we do not discuss here.

\section{One-loop scalar masses and the vacuum energy}

In the previous Section we have described a pair of type-I compactifications obtained by Scherk-Schwarz deformations of momenta and windings. Since in both models supersymmetry is broken, their spectra receive loop corrections. In particular, we are interested in the loop corrections to the vacuum energy and to the masses of the states unaffected by supersymmetry breaking. The M-theory breaking model is particularly interesting in this respect, since the residual global supersymmetry of its massless gauge sector is expected to be broken only by gravitational interactions in the small radius limit.

In this Section we compute the one-loop mass corrections, in the limit of small supersymmetry breaking, for the internal scalar components of ten-dimensional gauge fields, that remain massless to lowest order. Gauge invariance simplifies this task considerably: it makes these corrections universal, and allows one to deduce them from the potential induced by generic Wilson lines. We thus confine our attention to the scalar modes in the Cartan subalgebra, whose (constant) VEV's can be identified with open-string Wilson lines. In the supersymmetric case, these define flat directions in the theory. In the broken case, although they remain flat at the tree level, quantum corrections are expected to generate a potential, and we shall see that this is actually the case. Our method then consists in computing the partition function (cosmological constant) in the presence of Wilson lines, 
from which the masses can be obtained by differentiation at the origin 9 .

The one-loop vacuum energy results from the contributions of the four surfaces of vanishing Euler character:

$$
\begin{aligned}
\Lambda(W, R) & =\frac{1}{2} \int_{\mathcal{F}} \frac{d^{2} \tau}{\tau_{2}^{11 / 2}} \frac{\mathcal{T}(R)}{|\eta(\tau)|^{14}}+\int_{0}^{\infty} \frac{d \tau_{2}}{\tau_{2}^{11 / 2}} \frac{\mathcal{K}(R)}{\eta\left(2 i \tau_{2}\right)^{7}} \\
& +\int_{0}^{\infty} \frac{d t}{t^{11 / 2}} \frac{\mathcal{A}(W, R)}{\eta(i t / 2)^{7}}+\int_{0}^{\infty} \frac{d t}{t^{11 / 2}} \frac{\mathcal{M}(W, R)}{\hat{\eta}(i t / 2+1 / 2)^{7}}
\end{aligned}
$$

where $\mathcal{F}$ is the fundamental domain of the modular group for the world-sheet torus, and for the Klein-bottle the modulus $t$ is the $\tau_{2}$ of the previous Section. Furthermore, $W$ denotes a generic Wilson line affecting the open sector and $R$ denotes the radius of the circle. Referring to the two models of the previous Section, the limit of small supersymmetry breaking corresponds in the first case to $R \rightarrow \infty$, and in the second to $R \rightarrow 0$. The torus contribution behaves as $1 / R^{9}$ in the first case and as $R^{9}$ in the second 0 . Therefore, in the following we confine our attention to the remaining three contributions. The scalar masses may be obtained differentiating $\Lambda$ at the origin:

$$
m_{0}^{2}=\left.R^{2} \frac{\partial^{2} \Lambda(W)}{\partial a \partial a}\right|_{a=0}
$$

where the factor $R^{2}$ is due to the normalization of the kinetic terms for the scalar fields $a$ associated to the Wilson lines.

\subsection{Scherk-Schwarz breaking model}

Without loss of generality, in this case we can start from the model with an unbroken $S O(32)$ gauge group and introduce the Wilson line $W=\operatorname{diag}\left(e^{2 \pi i a_{1}}, e^{-2 \pi i a_{1}}, \cdots\right.$ $\left.e^{2 \pi i a_{16}}, e^{-2 \pi i a_{16}}\right)$. The Klein bottle is not affected [17], while the corresponding annulus

\footnotetext{
${ }^{9}$ Other nearby choices for the vacuum would generically supplement the soft masses with supersymmetric contributions.

${ }^{10}$ For reductions to $d$ non-compact dimensions, they would behave as $1 / R^{d}$ and as $R^{d}$, respectively.
} 
and Möbius direct-channel amplitudes are given in eq. (5.31). In the transverse channel representation, one then finds

$$
\begin{aligned}
\tilde{\mathcal{A}}_{1} & =\frac{2^{-11 / 2}}{2} R \sum_{n}\left[\left(V_{8}-S_{8}\right)\left(\operatorname{Tr} W^{2 n}\right)^{2} \tilde{Z}_{2 n}+\left(I_{8}-C_{8}\right)\left(\operatorname{Tr} W^{2 n+1}\right)^{2} \tilde{Z}_{2 n+1}\right], \\
\tilde{\mathcal{M}}_{1} & =-\frac{R}{\sqrt{2}} \sum_{n}\left[\hat{V}_{8}-(-1)^{n} \hat{S}_{8}\right]\left(\operatorname{Tr} W^{2 n}\right) \tilde{Z}_{2 n} .
\end{aligned}
$$

It is convenient to express these results in terms of the cylinder length $l$, related to the direct-channel variables as in eqs. (5.3) and (5.6). Aside from the torus contribution, the cosmological constant then reads:

$$
\Lambda_{\text {extra }}(W, R)=\int_{0}^{\infty} d l\left(\frac{\tilde{\mathcal{K}}_{1}(R)}{\eta(i l)^{7}}+\frac{\tilde{\mathcal{A}}_{1}(W, R)}{\eta(i l)^{7}}+\frac{\tilde{\mathcal{M}}_{1}(W, R)}{\hat{\eta}(i l+1 / 2)^{7}}\right) .
$$

In these models we are interested in the $R \rightarrow \infty$ limit, where the dominant contribution comes from the $l \rightarrow 0$, infrared region of integration. Using the "abstruse identity" $V_{8}=S_{8}$, the contribution of the Klein bottle vanishes. On the other hand, the annulus and Möbius transverse-channel amplitudes take the form

$$
\begin{aligned}
\int_{0}^{\infty} d l \frac{\tilde{\mathcal{A}}_{1}}{\eta(i l)^{7}} & =\frac{R}{2^{6} \sqrt{2}} \int_{0}^{\infty} d l \frac{\theta_{4}^{4}}{\eta^{12}}(i l) \sum_{n}\left(\operatorname{Tr} W^{2 n+1}\right)^{2} e^{-\frac{\pi l(2 n+1)^{2} R^{2}}{4}} \\
\int_{0}^{\infty} d l \frac{\tilde{\mathcal{M}}_{1}}{\hat{\eta}(i l+1 / 2)^{7}} & =-\frac{R}{\sqrt{2}} \int_{0}^{\infty} d l \frac{\hat{\theta}_{2}^{4}}{\hat{\eta}^{12}}\left(i l+\frac{1}{2}\right) \sum_{n}\left(\operatorname{Tr} W^{2(2 n+1)}\right) e^{-\pi l(2 n+1)^{2} R^{2}},
\end{aligned}
$$

and in the large-radius limit have the asymptotic behavior

$$
\begin{aligned}
\int_{0}^{\infty} d l \frac{\tilde{\mathcal{A}}_{1}}{\eta(i l)^{7}} & \underset{R \rightarrow \infty}{\sim} \frac{1}{4 R^{9} \sqrt{2}} \int_{0}^{\infty} d l l^{4} \sum_{n}\left(\operatorname{Tr} W^{2 n+1}\right)^{2} e^{-\frac{\pi l(2 n+1)^{2}}{4}} \\
\int_{0}^{\infty} d l \frac{\tilde{\mathcal{M}}_{1}}{\hat{\eta}(i l+1 / 2)^{7}} & \underset{R \rightarrow \infty}{\sim}-\frac{2^{8}}{R^{9} \sqrt{2}} \int_{0}^{\infty} d l l^{4} \sum_{n}\left(\operatorname{Tr} W^{2(2 n+1)}\right) e^{-\pi l(2 n+1)^{2}} .
\end{aligned}
$$

In deriving the last expressions, we have rescaled the integration variable according to $l \rightarrow$ $l / R^{2}$, and we have extracted, after a modular transformation, the dominant contribution of the string oscillators as $R \rightarrow \infty$.

The integrals over $l$ are convergent and the result is

$$
\Lambda_{\text {extra }} \simeq \frac{2^{11} 3}{\sqrt{2} \pi^{5} R^{9}} \sum_{n} \frac{1}{(2 n+1)^{10}}\left[\left(\operatorname{Tr} W^{2 n+1}\right)^{2}-\operatorname{Tr} W^{2(2 n+1)}\right] .
$$


From eq. (6.2) the soft scalar masses then behave as $1 / R^{7 / 2}$ in the large radius limit. As $W \rightarrow 1$, the factor tend to the usual field theory factor, proportional to the number of particles circulating in the loop. More generally, if the theory were compactified to $d$ dimensions, one would obtain a vacuum energy $\Lambda$ scaling as $1 / R^{d}$ and scalar masses scaling as $1 / R^{(d-2) / 2}$. Therefore, in compactifications to four dimensions all soft masses are of the same order of magnitude

$$
m_{0} \sim m_{1 / 2} \sim m_{3 / 2} \sim \frac{1}{R}
$$

as expected from the effective field theory.

\subsection{M-theory breaking model}

Strictly speaking, in this case Wilson lines are not allowed. This was pointed out in [9] for the supersymmetric model, where it was argued that the dilaton would otherwise develop strong coupling singularities. We shall see shortly a different manifestation of the same

phenomenon: a divergent tadpole appears in the $R \rightarrow 0$ limit. This applies also to the Scherk-Schwarz deformed model. With these qualifications, we turn on two sets of Wilson lines $W$ and $W^{\prime}$ in the two factors of the gauge group. The direct-channel annulus and Möbius amplitudes (5.39) then become

$$
\begin{aligned}
\mathcal{A}_{2} & =\frac{1}{2}\left(V_{8}-S_{8}\right) \sum_{i, j=1}^{16}\left(Z_{2\left(m+a_{i}+a_{j}\right)}+Z_{2\left(m+a_{i}^{\prime}+a_{j}^{\prime}\right)}\right)+\frac{1}{2}\left(I_{8}-C_{8}\right) \sum_{i, j=1}^{16} Z_{2\left(m+1 / 2+a_{i}+a_{j}^{\prime}\right)}, \\
\mathcal{M}_{2} & =-\frac{1}{2}\left[\hat{V}_{8}-(-1)^{m} \hat{S}_{8}\right] \sum_{i=1}^{16}\left(Z_{2\left(m+2 a_{i}\right)}+Z_{2\left(m+2 a_{i}^{\prime}\right)}\right)
\end{aligned}
$$

while in the transverse channel the various amplitudes read

$$
\begin{aligned}
\tilde{\mathcal{K}}_{2} & =\frac{2^{9 / 2}}{2} R\left(V_{8} \tilde{Z}_{2 n}-S_{8} \tilde{Z}_{2 n+1}\right) \\
\tilde{\mathcal{A}}_{2} & =\frac{2^{-11 / 2}}{2} R\left[V_{8} \sum_{n}\left(\operatorname{Tr} W^{n}+(-1)^{n} \operatorname{Tr} W^{\prime n}\right)^{2} \tilde{Z}_{n}-S_{8}\left(\operatorname{Tr} W^{n}-(-1)^{n} \operatorname{Tr} W^{\prime n}\right)^{2} \tilde{Z}_{n}\right] \\
\tilde{\mathcal{M}}_{2} & =-\frac{R}{\sqrt{2}}\left[\hat{V}_{8} \sum_{n}\left(\operatorname{Tr} W^{2 n}+\operatorname{Tr} W^{\prime 2 n}\right) \tilde{Z}_{2 n}-\hat{S}_{8}\left(\operatorname{Tr} W^{2 n+1}+\operatorname{Tr} W^{2 n+1}\right) \tilde{Z}_{2 n+1}\right]
\end{aligned}
$$


In this model we are interested in the $R \rightarrow 0$ limit. This is dominated by the $l \rightarrow \infty$, ultraviolet region of integration, and is rather subtle, since as $R \rightarrow 0$ all winding states flowing in the transverse channel contribute to massless tadpoles. The tadpole conditions then read

$$
\operatorname{Tr} W^{2 n}=\operatorname{Tr} W^{\prime 2 n}=16, \quad \operatorname{Tr} W^{2 n+1}=\operatorname{Tr} W^{2 n+1}=16
$$

and demand that $W=W^{\prime}=1$, so that the $S O(16) \times S O(16)$ gauge group is to remain unbroken. In fact, the same conditions apply to the supersymmetric model, and provide a different manifestation of the phenomenon first noticed in [9]. In the type- $\mathrm{I}^{\prime}$ language, this limit would naively correspond to weak coupling, but the dilaton actually develops strongcoupling singularities at particular points on the circle 9]. From this perspective, the pathologically large vacuum values of the dilaton are clearly induced by the new tadpoles resulting from the collapsed massive states. Therefore, a proper perturbative definition of the theory requires that all the conditions (6.11) be satisfied.

After the rescaling $l \rightarrow l / R^{2}$, in the $R \rightarrow 0$ limit the transverse amplitudes read

$$
\begin{aligned}
\int_{0}^{\infty} d l \frac{\tilde{\mathcal{K}}_{2}}{\eta(i l)^{7}} & =\frac{2^{9 / 2}}{2 R} \int_{0}^{\infty} d l \frac{\theta_{2}^{4}}{2 \eta^{12}}\left(i l / R^{2}\right)(-1)^{n} e^{-\frac{\pi l n^{2}}{4}}, \\
\int_{0}^{\infty} d l \frac{\tilde{\mathcal{A}}_{2}}{\eta(i l)^{7}} & =\frac{2^{-7 / 2}}{2 R} \int_{0}^{\infty} d l \frac{\theta_{2}^{4}}{2 \eta^{12}}\left(i l / R^{2}\right)(-1)^{n} e^{-\frac{\pi l n^{2}}{4}} \operatorname{Tr} W^{n} \operatorname{Tr} W^{\prime n}, \\
\int_{0}^{\infty} d l \frac{\tilde{\mathcal{M}}_{2}}{\hat{\eta}(i l+1 / 2)^{7}} & =-\frac{1}{\sqrt{2} R} \int_{0}^{\infty} d l \frac{\hat{\theta}_{2}^{4}}{2 \hat{\eta}^{12}}\left(i l / R^{2}+1 / 2\right)(-1)^{n} e^{-\frac{\pi l n^{2}}{4}}\left(\operatorname{Tr} W^{n}+\operatorname{Tr} W^{\prime n}\right),
\end{aligned}
$$

where we have used the "abstruse identity" $V_{8}=S_{8}=\theta_{2}^{4} / 2 \eta^{4}$. Moreover, in this limit only the ground state contributes. Its degeneracy can simply be accounted for letting $S_{8} / \eta^{8}, \hat{S}_{8} / \hat{\eta}^{8} \rightarrow 8$, and thus

$$
\begin{gathered}
\Lambda_{\text {extra }} \underset{R \rightarrow 0}{\sim} \frac{1}{2 \sqrt{2} R} \int_{0}^{\infty} d l \sum_{n} \operatorname{Tr}\left(W^{n}-1\right) \operatorname{Tr}\left(W^{\prime n}-1\right)(-1)^{n} e^{-\frac{\pi l n^{2}}{4}} \\
\quad=\frac{\sqrt{2}}{\pi R} \sum_{n} \frac{(-1)^{n}}{n^{2}} \operatorname{Tr}\left(W^{n}-1\right) \operatorname{Tr}\left(W^{\prime n}-1\right) .
\end{gathered}
$$

Therefore, the leading contribution to the potential $\Lambda_{\text {extra }}$ is quartic in the Wilson lines and the soft scalar masses vanish in this approximation, up to exponentially suppressed 
corrections $\mathcal{O}(\exp (-\pi / R))$. The latter come from the massive oscillator modes of the string, that we have neglected. Furthermore, in this limit the one-loop vacuum energy of the M-theory breaking model is not corrected to lowest order by $\Lambda_{\text {extra }}$ and is given by the torus contribution, that behaves as $R^{9}$. This result implies that the soft scalar masses are dominated by gravitational contributions, that first arise from genus $3 / 2$ world-sheets, and by field theory considerations are expected to give a contribution suppressed by powers of the Planck mass.

\section{Supersymmetry breaking in six dimensions}

In Section 5 we have described two distinct 9d models with supersymmetry breaking induced by Scherk-Schwarz deformations affecting the momenta or the windings. The generalization to lower dimensions involves, in general, the simultaneous presence of D9 and D5 branes (modulo T-dualities). When only one type of brane is present, the previous construction admits a straightforward generalization that follows directly from the corresponding deformation of the torus amplitude. The purpose of this Section is to illustrate a simple instance of supersymmetry breaking in six dimensions in the presence of D9 and D5 branes. We should emphasize, however, that in this model the Scherk-Schwarz deformation is accompanied by a subtle change of chirality for the twisted fermions already in the closed sector. This phenomenon, peculiar to six dimensions, obscures somewhat the spontaneous nature of the breaking mechanism.

In six dimensions, supersymmetric open-string spectra have a rich structure, due to the possible presence of D9 and D5 branes [15, 20, 21]. By heterotic-type I duality, nine branes correspond to perturbative gauge groups on the heterotic side, while five-branes correspond to zero-size heterotic instantons. As nine branes and five branes are T-dual of each other, the two basic ways of breaking supersymmetry discussed in Sections 4 and 5, with soft masses proportional to $1 / R$ and to $R$, are expected to combine. In particular, the previous 
examples suggest that only one massless sector (the 99 one) feels supersymmetry breaking at tree level, while the other massless sectors (the 59 and the 55 ones) can only be affected via radiative corrections.

In order to discuss the breaking of $(1,0)$ supersymmetry in $6 \mathrm{~d}$, we can start from a sixdimensional IIB orbifold model and perform a Scherk-Schwarz deformation of the closed string partition function in a way compatible with modular invariance, particle interpretation and the orbifold projection. The original IIB massless spectrum has $(2,0)$ supersymmetry, and is uniquely fixed by anomaly cancellation: it consists of the gravitational multiplet, with $\left(g_{\mu \nu}, 2 \psi_{\mu L}, 5 B_{\mu \nu}^{+}\right)$and of 21 tensor multiplets, with $\left(B_{\mu \nu}^{-}, 2 \chi_{R}, 5 \phi\right)$, where $B^{+}\left(B^{-}\right)$denote tensors with (anti)self-dual field strengths and the chirality of the fermionic fields is indicated by the $L$ and $R$ subscripts. The corresponding open descendants have $(1,0)$ supersymmetry and, in addition to the gravitational multiplet $\left(g_{\mu \nu}, \psi_{\mu L}, B_{\mu \nu}^{+}\right)$, contain in general variable numbers of tensor multiplets $\left(B_{\mu \nu}^{-}, \chi_{R}, \phi\right)$, vector multiplets $\left(A_{\mu}, \lambda_{L}\right)$ and hypermultiplets $\left(\psi_{R}, 4 \phi\right)$.

To be concrete, let us consider in detail the $T^{4} / Z_{2}$ type I orbifold of [15, 20] with all five-branes at the same fixed point, with a single tensor multiplet, and with gauge group $U(16)_{9} \times U(16)_{5}$. The open string sector then contains vector multiplets and hypermultiplets in the representations $(\mathbf{1 2 0}+\overline{\mathbf{1 2 0}}, \mathbf{1}),(\mathbf{1}, \mathbf{1 2 0}+\overline{\mathbf{1 2 0}})$ of the gauge group from the 99 and 55 sectors, and one hypermultiplet in the representation $(\mathbf{1 6}, \mathbf{1 6})$ from the 59 sector.

Following [15], let us introduce the convenient combinations of $S O(4)$ characters

$$
\begin{aligned}
& Q_{O}=V_{4} I_{4}-C_{4} C_{4}, \quad Q_{V}=I_{4} V_{4}-S_{4} S_{4}, \\
& Q_{S}=I_{4} C_{4}-S_{4} I_{4},
\end{aligned}
$$

that allow to write the partition function in a compact form. By definition, the first factor in (7.1) refers to the transverse coordinates of spacetime, while the second refers to the internal (compact) ones. Thus, for instance, $Q_{O}$ describes a Neveu-Schwarz vector and a corresponding Ramond $L$ spinor, $Q_{V}$ describes 4 scalars and a corresponding $R$ spinor, while 
the relative sign between bosonic and fermionic contributions is dictated by spin statistics. In terms of these characters, the torus amplitude of the $N=(2,0)$ supersymmetric $T^{4} / Z_{2}$ orbifold reads

$$
\begin{aligned}
\mathcal{T} & =\frac{1}{2} \Lambda^{(4,4)}\left|V_{8}-S_{8}\right|^{2}+\frac{1}{2}\left|Q_{O}-Q_{V}\right|^{2}\left|I_{4} I_{4}-V_{4} V_{4}\right|_{B}^{2} \\
& +\frac{1}{2}\left\{\left|Q_{S}+Q_{C}\right|^{2}\left|Q_{S}+Q_{C}\right|_{B}^{2}+\left|Q_{S}-Q_{C}\right|^{2}\left|Q_{S}-Q_{C}\right|_{B}^{2}\right\}
\end{aligned}
$$

where $\Lambda^{(4,4)}$ denotes a $\Gamma_{(4,4)}$ Narain lattice [19] for the compact coordinates with a vanishing antisymmetric tensor, and the subscript $B$ refers to the compact bosonic modes, fermionized according to

$$
\begin{aligned}
4 \frac{\eta^{2}}{\theta_{2}^{2}} & =\frac{\theta_{3}^{2} \theta_{4}^{2}}{\eta^{4}}=\left(I_{4} I_{4}-V_{4} V_{4}\right)_{B} \quad, \quad 4 \frac{\eta^{2}}{\theta_{4}^{2}}=\frac{\theta_{2}^{2} \theta_{3}^{2}}{\eta^{4}}=\left(Q_{S}+Q_{C}\right)_{B} \\
4 \frac{\eta^{2}}{\theta_{3}^{2}} & =\frac{\theta_{2}^{2} \theta_{4}^{2}}{\eta^{4}}=\left(Q_{S}-Q_{C}\right)_{B} .
\end{aligned}
$$

We can now break supersymmetry deforming the partition function along a compact direction. To this end, let us specialize to a factorized $\Gamma_{(1,1)} \times \Gamma_{(3,3)}$ lattice and deform the first term of eq. (7.2) according to (4.6) with $\mathbf{e}=(0,0,0,1)$. This corresponds to the operator $(-1)^{F}$ acting on the lattice states, and results from a $2 \pi$-rotation in a plane defined by one compact and one non-compact coordinate. The corresponding world-sheet current anticommutes with the orbifold projection, as required for the consistency of the construction [5, 1]. It is also convenient to introduce the additional characters

$$
\begin{aligned}
& Q_{O}^{\prime}=V_{4} I_{4}-S_{4} S_{4}, \quad Q_{V}^{\prime}=I_{4} V_{4}-C_{4} C_{4}, \\
& Q_{S}^{\prime}=I_{4} S_{4}-C_{4} I_{4}, \quad Q_{C}^{\prime}=V_{4} C_{4}-S_{4} V_{4},
\end{aligned}
$$

so that the deformed partition function reads

$$
\begin{aligned}
& \mathcal{T}=\frac{\Lambda^{(3,3)}}{2}\left\{E_{0}^{\prime}\left(\left|V_{8}\right|^{2}+\left|S_{8}\right|^{2}\right)+O_{0}^{\prime}\left(\left|I_{8}\right|^{2}+\left|C_{8}\right|^{2}\right)-E_{1 / 2}^{\prime}\left(V_{8} \bar{S}_{8}+S_{8} \bar{V}_{8}\right)-O_{1 / 2}^{\prime}\left(I_{8} \bar{C}_{8}+C_{8} \bar{I}_{8}\right)\right\} \\
& +\frac{1}{4}\left(\left|Q_{O}-Q_{V}\right|^{2}+\left|Q_{O}^{\prime}-Q_{V}^{\prime}\right|^{2}\right)\left|I_{4} I_{4}-V_{4} V_{4}\right|_{B}^{2} \\
& +\frac{1}{4}\left\{\left(\left|Q_{S}+Q_{C}\right|^{2}+\left|Q_{S}^{\prime}+Q_{C}^{\prime}\right|^{2}\right)\left|Q_{S}+Q_{C}\right|_{B}^{2}+\left(\left|Q_{S}-Q_{C}\right|^{2}+\left|Q_{S}^{\prime}-Q_{C}^{\prime}\right|^{2}\right)\left|Q_{S}-Q_{C}\right|_{B}^{2}\right\} .
\end{aligned}
$$


Compared to the supersymmetric case (7.2), in the untwisted sector of the deformed closed string (7.5) all fermion masses are shifted by $1 /(2 R)$. On the other hand, as expected, in the twisted sector the fermion masses are unchanged, while the chirality of half of the fermions is inverted. Eight of the twisted massless fermions are thus left handed, while the other eight are right handed. This phenomenon will have a counterpart in the open sector. It originates from the modifications introduced by the Scherk-Schwarz breaking at the origin of the lattice, that eliminate all the corresponding fermionic modes. This affects the orbifold projection in the untwisted sector and, by modular invariance, modifies the chirality of the twisted states. This phenomenon is peculiar to six-dimensional models, where closed string states can carry a net chirality, in contrast to the more familiar four-dimensional case.

Following the same steps as in Section 5, the Klein bottle amplitudes in the direct and transverse channels are

$$
\begin{aligned}
\mathcal{K} & =\frac{1}{4}\left[\left(V_{8}-S_{8}\right)\left(Z_{m} \Lambda^{(3)}+\tilde{Z}_{2 n} \tilde{\Lambda}^{(3)}\right)+\left(I_{8}-C_{8}\right) \tilde{Z}_{2 n+1} \tilde{\Lambda}^{(3)}+\left(Q_{S}+Q_{C}+Q_{S}^{\prime}+Q_{C}^{\prime}\right)\left(Q_{S}+Q_{C}\right)_{B}\right] \\
\tilde{\mathcal{K}} & =\frac{2^{5}}{4}\left[v_{4}\left(V_{8}-S_{8}\right) \tilde{Z}_{2 n} \tilde{\Lambda}_{e}^{(3)}+\frac{1}{v_{4}}\left(V_{8} Z_{2 m}-S_{8} Z_{2 m+1}\right) \Lambda_{e}^{(3)}\right] \\
& +\frac{2^{5}}{4}\left(Q_{O}-Q_{V}+Q_{O}^{\prime}-Q_{V}^{\prime}\right)\left(I_{4} I_{4}-V_{4} V_{4}\right)_{B}
\end{aligned}
$$

where $v_{4}$ is the volume of the compact space, $\Lambda^{(3)}$ contains only momenta and $\tilde{\Lambda}^{(3)}$ contains only windings. Moreover, in $\Lambda_{e}^{(3)}$ and $\tilde{\Lambda}_{e}^{(3)}$ the lattice sums are restricted to even values of momenta and windings, respectively.

It is instructive to exhibit in $\tilde{\mathcal{K}}$ the general structure of eq. (5.4). While evident for the contributions of all lattice points away from the origin, this is not apparent for the remaining states. To this end, let us recall the decomposition of level-one $S O(8)$ characters into $S O(4)$ ones ,

$$
\begin{array}{ll}
I_{8}=I_{4} I_{4}+V_{4} V_{4}, & C_{8}=S_{4} C_{4}+C_{4} S_{4}, \\
V_{8}=V_{4} I_{4}+I_{4} V_{4}, & S_{8}=S_{4} S_{4}+C_{4} C_{4},
\end{array}
$$


that may be simply induced from elementary group embeddings. Leaving aside all terms away from the origin of the lattice, $\tilde{\mathcal{K}}$ reduces to

$$
\begin{aligned}
\tilde{\mathcal{K}}_{0} & =\frac{2^{5}}{4}\left[v_{4}\left(V_{4} I_{4}+I_{4} V_{4}-S_{4} S_{4}-C_{4} C_{4}\right)+\frac{1}{v_{4}}\left(V_{4} I_{4}+I_{4} V_{4}\right)\right]\left(I_{4} I_{4}+V_{4} V_{4}\right)_{B} \\
& +\frac{2^{5}}{4} 2\left(V_{4} I_{4}-I_{4} V_{4}\right)\left(I_{4} I_{4}-V_{4} V_{4}\right)_{B},
\end{aligned}
$$

where in the first term $\left(I_{4} I_{4}+V_{4} V_{4}\right)_{B}$ denotes the contribution from the origin of the Narain lattice. This expression can then be reassembled in the form

$$
\begin{aligned}
\tilde{\mathcal{K}}_{0} & =\frac{2^{5}}{4}\left(\sqrt{v}_{4}+\frac{1}{\sqrt{v}_{4}}\right)^{2}\left[V_{4} I_{4}\left(I_{4} I_{4}\right)_{B}+I_{4} V_{4}\left(V_{4} V_{4}\right)_{B}\right] \\
& +\frac{2^{5}}{4}\left(\sqrt{v}_{4}-\frac{1}{\sqrt{v}_{4}}\right)^{2}\left[V_{4} I_{4}\left(V_{4} V_{4}\right)_{B}+I_{4} V_{4}\left(I_{4} I_{4}\right)_{B}\right] \\
& -\frac{2^{5}}{4}\left(\sqrt{v}_{4}\right)^{2}\left(S_{4} S_{4}+C_{4} C_{4}\right)\left(I_{4} I_{4}+V_{4} V_{4}\right)_{B}
\end{aligned}
$$

where all coefficients are indeed perfect squares.

As in Section 5, the transverse-channel annulus amplitude results from contributions of the different sectors of the closed string, each weighted by a corresponding (squared) reflection coefficient. The non-vanishing coefficients pertain to the twisted states, as well as to the untwisted ones belonging to two sublattices of the original Narain lattice. In particular, terms with zero momentum correspond to Neumann boundary conditions, while terms with zero winding correspond to Dirichlet ones. These will shortly pair with corresponding terms in $\tilde{\mathcal{K}}$ proportional to the internal volume $v_{4}$ and to its inverse. Furthermore, the reflection coefficients are parametrized, as usual, in terms of some integers that we shall shortly relate to Chan-Paton multiplicities. For untwisted closed-string states, these comprise $n_{N}$, the dimensionality of the Chan-Paton space for the (Neumann) 9-brane charges and $\left(n_{D_{1}}, n_{D_{2}}\right)$, the corresponding dimensionalities for two types of (Dirichlet) 5-brane charges. In addition, the reflection coefficients for twisted closed-string states are parametrized in terms of the integers $R_{N}, R_{D_{1}}$ and $R_{D_{2}}$ [14. One thus finds:

$$
2^{7} \tilde{\mathcal{A}}=v_{4} n_{N}^{2}\left(\left(V_{8}-S_{8}\right) \tilde{Z}_{2 n}+\left(I_{8}-C_{8}\right) \tilde{Z}_{2 n+1}\right) \tilde{\Lambda}^{(3)}
$$




$$
\begin{aligned}
& +\frac{1}{v_{4}}\left(\left(n_{D_{1}}+n_{D_{2}}\right)^{2}\left(V_{8} Z_{2 m}-S_{8} Z_{2 m+1}\right)+\left(n_{D_{1}}-n_{D_{2}}\right)^{2}\left(V_{8} Z_{2 m+1}-S_{8} Z_{2 m}\right)\right) \Lambda^{(3)} \\
& +\left(2 n_{N} n_{D_{1}}\left(Q_{O}^{\prime}-Q_{V}^{\prime}\right)+2 n_{N} n_{D_{2}}\left(Q_{O}-Q_{V}\right)\right)\left(I_{4} I_{4}-V_{4} V_{4}\right)_{B} \\
& +\left(2 R_{N}^{2}\left(Q_{S}+Q_{C}+Q_{S}^{\prime}+Q_{C}^{\prime}\right)+4 R_{D_{2}}^{2}\left(Q_{S}+Q_{C}\right)+4 R_{D_{1}}^{2}\left(Q_{S}^{\prime}+Q_{C}^{\prime}\right)\right)\left(Q_{S}+Q_{C}\right)_{B} \\
& +\left(2 R_{N} R_{D_{2}}\left(Q_{S}-Q_{C}\right)+2 R_{N} R_{D_{1}}\left(Q_{S}^{\prime}-Q_{C}^{\prime}\right)\right)\left(Q_{S}-Q_{C}\right)_{B}
\end{aligned}
$$

Just like $\tilde{\mathcal{K}}$, the transverse-channel annulus amplitude $\tilde{\mathcal{A}}$ satisfies a rather stringent consistency condition: the reflection coefficients of the various sectors of the closed spectrum are to be perfect squares, as in eq. (5.5). Again, this property is not apparent for the contributions arising from the origin of the Narain lattice and from twisted sectors. It is therefore instructive to display these terms, that we denote collectively by $\tilde{\mathcal{A}}_{0}$, in the properly reassembled form:

$$
\begin{aligned}
2^{7} \tilde{\mathcal{A}}_{0} & =\left(\sqrt{v_{4}} n_{N}+\frac{n_{D_{1}}+n_{D_{2}}}{\sqrt{v}_{4}}\right)^{2}\left[V_{4} I_{4}\left(I_{4} I_{4}\right)_{B}+I_{4} V_{4}\left(V_{4} V_{4}\right)_{B}\right] \\
& +\left(\sqrt{v_{4}} n_{N}-\frac{n_{D_{1}}+n_{D_{2}}}{\sqrt{v}}\right)^{2}\left[V_{4} I_{4}\left(V_{4} V_{4}\right)_{B}+I_{4} V_{4}\left(I_{4} I_{4}\right)_{B}\right] \\
& -\left(\sqrt{v_{4}} n_{N}+\frac{n_{D_{1}}-n_{D_{2}}}{\sqrt{v}_{4}}\right)^{2}\left[S_{4} S_{4}\left(I_{4} I_{4}\right)_{B}+C_{4} C_{4}\left(V_{4} V_{4}\right)_{B}\right] \\
& -\left(\sqrt{v_{4}} n_{N}-\frac{n_{D_{1}}-n_{D_{2}}}{\sqrt{v}_{4}}\right)^{2}\left[S_{4} S_{4}\left(V_{4} V_{4}\right)_{B}+C_{4} C_{4}\left(I_{4} I_{4}\right)_{B}\right] \\
& +\left(\frac{\left(R_{N}+4 R_{D_{2}}\right)^{2}}{4}+\frac{7 R_{N}^{2}}{4}\right)\left(Q_{S} Q_{S B}+Q_{C} Q_{C B}\right) \\
& +\left(\frac{\left(R_{N}-4 R_{D_{2}}\right)^{2}}{4}+\frac{7 R_{N}^{2}}{4}\right)\left(Q_{S} Q_{C B}+Q_{C} Q_{S B}\right) \\
& +\left(\frac{\left(R_{N}+4 R_{D_{1}}\right)^{2}}{4}+\frac{7 R_{N}^{2}}{4}\right)\left(Q_{S}^{\prime} Q_{S B}+Q_{C}^{\prime} Q_{C B}\right) \\
& +\left(\frac{\left(R_{N}-4 R_{D_{1}}\right)^{2}}{4}+\frac{7 R_{N}^{2}}{4}\right)\left(Q_{S}^{\prime} Q_{C B}+Q_{C}^{\prime} Q_{S B}\right) .
\end{aligned}
$$

The last four lines are particularly interesting, since they describe the reflections of the twisted sectors of the closed string. As is well known, these are confined to the 16 fixed points of the orbifold, and thus eq. (7.11) contains a rather detailed information on the 
geometry of the D5 branes. There is a little complication, however, since we are actually describing the full twisted sector in terms of two pairs of characters $\left(Q_{S}, Q_{C}\right)$ and $\left(Q_{S}^{\prime}, Q_{C}^{\prime}\right)$. While these suffice to identify two types of fixed points, they clearly cannot distinguish any two fixed points of the same type. As a result, in this case the reflection coefficients are actually sums of squares. Still, their interpretation is quite transparent. The terms related to the pair $\left(Q_{S}, Q_{C}\right)$ imply that all D5 branes of one type are concentrated in one of eight available fixed points, and the terms related to the pair $\left(Q_{S}^{\prime}, Q_{C}^{\prime}\right)$ imply a similar distribution for the second type of D5 branes among the remaining eight fixed points. To reiterate, out of the 16 fixed points of the orbifold 14 are empty, and the corresponding sectors of the closed string sense only the ubiquitous nine-branes. One of the remaining two fixed points accommodates all the D5 branes of one type, while the other accommodates all those of the second type. Actually, as we shall see shortly, the tadpole conditions imply that the model includes a total of 16 D5 branes, as does the standard version with unbroken supersymmetry, so that eight of them are actually located at each of these two fixed points.

A modular $S$ transformation determined by eq. (5.14) yields the direct-channel annulus amplitude

$$
\begin{aligned}
\mathcal{A} & \left.=\frac{n_{N}^{2}}{4}\left(V_{8} Z_{2 m}-S_{8} Z_{2(m+1 / 2)}\right) \Lambda^{(3)}+\frac{1}{4}\left(n_{D_{1}}^{2}+n_{D_{2}}^{2}\right)\left(V_{8}-S_{8}\right) \tilde{Z}_{2 n}+2 n_{D_{1}} n_{D_{2}}\left(I_{8}-C_{8}\right) \tilde{Z}_{2(n+1 / 2)}\right) \tilde{\Lambda}_{0}^{(3)} \\
& +\frac{1}{4}\left(2 n_{N} n_{D_{1}}\left(Q_{S}^{\prime}+Q_{C}^{\prime}\right)+2 n_{N} n_{D_{2}}\left(Q_{S}+Q_{C}\right)\right)\left(\frac{Q_{S}+Q_{C}}{4}\right)_{B} \\
& +\frac{1}{4}\left(\frac{1}{2} R_{N}^{2}\left(Q_{O}-Q_{V}+Q_{O}^{\prime}-Q_{V}^{\prime}\right)+R_{D_{2}}^{2}\left(Q_{O}-Q_{V}\right)+R_{D_{1}}^{2}\left(Q_{O}^{\prime}-Q_{V}^{\prime}\right)\right)\left(I_{4} I_{4}-V_{4} V_{4}\right)_{B} \\
& +\frac{1}{4}\left(2 R_{N} R_{D_{2}}\left(Q_{S}-Q_{C}\right)+2 R_{N} R_{D_{1}}\left(Q_{S}^{\prime}-Q_{C}^{\prime}\right)\right)\left(\frac{Q_{S}-Q_{C}}{4}\right)_{B} .
\end{aligned}
$$

As in [14, the $R$ terms describe the combined action of the orbifold involution on the openstring sectors and on the corresponding Chan-Paton charges. In our case, the orbifold involution consists of a pair of $\pi$-rotations in the $(6,7)$ and $(8,9)$ planes that split the contributions to eq. (7.7), inverting the signs of the terms involving the internal $V_{4}$ and $S_{4}$. The $R$ terms effect a corresponding splitting in the Chan-Paton charge space. There are 
actually two distinct options in this case, that correspond to "real" and "complex" charges or, equivalently, to orthogonal or symplectic and to unitary groups, respectively. In the former case, $N=n_{+}+n_{-}$, and

$$
R=n_{+}-n_{-} \quad
$$

where $n_{+}$and $n_{-}$are identified with the charge multiplicities in the unbroken gauge groups. In the latter case [15], $N=n+\bar{n}$, and

$$
R=i(n-\bar{n}) \quad
$$

where $n$ and $\bar{n}$ are identified with the (identical) charge multiplicities of the fundamental and conjugate representations of a unitary group. Indeed, one can simply see that in this case the positivity constraints on the transverse-channel annulus amplitude require that $n=\bar{n}$.

The geometric means of the reflection coefficients for the sectors common to $\tilde{\mathcal{K}}$ and $\tilde{\mathcal{A}}$ now determine the transverse-channel Möbius amplitude:

$$
\begin{aligned}
-2 \tilde{\mathcal{M}} & =n_{N} v_{4}\left(\hat{V}_{8}\left(\tilde{Z}_{4 n}+\tilde{Z}_{4 n+2}\right)-\hat{S}_{8}\left(\tilde{Z}_{4 n}-\tilde{Z}_{4 n+2}\right)\right) \tilde{\Lambda}_{e}^{(3)}+\frac{\left(n_{D_{1}}+n_{D_{2}}\right)}{v_{4}}\left(\hat{V}_{8} Z_{2 m}-\hat{S}_{8} Z_{2 m+1}\right) \Lambda_{e}^{(3)} \\
& +\left(n_{N}\left(V_{4} I_{4}-I_{4} V_{4}\right)+n_{D_{1}}\left(\hat{Q}_{O}^{\prime}-\hat{Q}_{V}^{\prime}\right)+n_{D_{2}}\left(\hat{Q}_{O}-\hat{Q}_{V}\right)\right)\left(\hat{I}_{4} \hat{I}_{4}-\hat{V}_{4} \hat{V}_{4}\right)_{B}
\end{aligned}
$$

The reader can easily verify that the terms at the origin of the lattice, $\tilde{\mathcal{M}}_{0}$, take the form of eq. (5.8). Indeed, starting from eq. (7.15) one gets

$$
\begin{aligned}
-2 \tilde{\mathcal{M}}_{0} & =\left(n_{N} v_{4}\left(\hat{V}_{8}-\hat{S}_{8}\right)+\frac{1}{v_{4}}\left(n_{D_{1}}+n_{D_{2}}\right) \hat{V}_{8}\right)\left(\hat{I}_{4} \hat{I}_{4}+\hat{V}_{4} \hat{V}_{4}\right)_{B} \\
& +\left(n_{N}\left(\hat{V}_{4} \hat{I}_{4}-\hat{I}_{4} \hat{V}_{4}\right)+n_{D_{1}}\left(\hat{Q}_{O}^{\prime}-\hat{Q}_{V}^{\prime}\right)+n_{D_{2}}\left(\hat{Q}_{O}-\hat{Q}_{V}\right)\right)\left(\hat{I}_{4} \hat{I}_{4}-\hat{V}_{4} \hat{V}_{4}\right)_{B}
\end{aligned}
$$

that, using the decompositions (7.7), becomes:

$$
\begin{aligned}
-2 \tilde{\mathcal{M}}_{0} & =\left(\sqrt{v}_{4}+\frac{1}{\sqrt{v}_{4}}\right)\left(\sqrt{v}_{4} n_{N}+\frac{n_{D_{1}}+n_{D_{2}}}{\sqrt{v}_{4}}\right)\left[\hat{V}_{4} \hat{I}_{4}\left(\hat{I}_{4} \hat{I}_{4}\right)_{B}+\hat{I}_{4} \hat{V}_{4}\left(\hat{V}_{4} \hat{V}_{4}\right)_{B}\right] \\
& +\left(\sqrt{v}_{4}-\frac{1}{\sqrt{v}_{4}}\right)\left(\sqrt{v}_{4} n_{N}-\frac{n_{D_{1}}+n_{D_{2}}}{\sqrt{v}_{4}}\right)\left[\hat{V}_{4} \hat{I}_{4}\left(\hat{V}_{4} \hat{V}_{4}\right)_{B}+\hat{I}_{4} \hat{V}_{4}\left(\hat{I}_{4} \hat{I}_{4}\right)_{B}\right]
\end{aligned}
$$




$$
\begin{aligned}
& -\sqrt{v}_{4}\left(\sqrt{v}_{4} n_{N}+\frac{n_{D_{1}}-n_{D_{2}}}{\sqrt{v}_{4}}\right)\left[\hat{S}_{4} \hat{S}_{4}\left(\hat{I}_{4} \hat{I}_{4}\right)_{B}+\hat{C}_{4} \hat{C}_{4}\left(\hat{V}_{4} \hat{V}_{4}\right)_{B}\right] \\
& -\sqrt{v}_{4}\left(\sqrt{v}_{4} n_{N}-\frac{n_{D_{1}}-n_{D_{2}}}{\sqrt{v}_{4}}\right)\left[\hat{S}_{4} \hat{S}_{4}\left(\hat{V}_{4} \hat{V}_{4}\right)_{B}+\hat{C}_{4} \hat{C}_{4}\left(\hat{I}_{4} \hat{I}_{4}\right)_{B}\right] .
\end{aligned}
$$

The $P_{(4)}$ matrix of eq. (5.14) then determines the direct channel Möbius amplitude :

$$
\begin{aligned}
\mathcal{M} & =-\frac{n_{N}}{4}\left(\hat{V}_{8} Z_{2 m}-\hat{S}_{8} Z_{2(m+1 / 2)}\right) \Lambda^{(3)}-\frac{n_{D_{1}}+n_{D_{2}}}{4}\left(\hat{V}_{8} \tilde{Z}_{2 n}-\hat{S}_{8}(-1)^{n} \tilde{Z}_{2 n}\right) \tilde{\Lambda}_{0}^{(3)} \\
& +\frac{1}{4}\left(n_{N}\left(V_{4} I_{4}-I_{4} V_{4}\right)+n_{D_{1}}\left(\hat{Q}_{O}^{\prime}-\hat{Q}_{V}^{\prime}\right)+n_{D_{2}}\left(\hat{Q}_{O}-\hat{Q}_{V}\right)\right)\left(\hat{I}_{4} \hat{I}_{4}-\hat{V}_{4} \hat{V}_{4}\right)_{B} .
\end{aligned}
$$

The tadpole equations related to twisted states demand that

$$
R_{N}=R_{D_{1}}=R_{D_{2}}=0
$$

since the corresponding characters appear only in the annulus. Three more tadpole conditions originate from the massless contributions of untwisted states, that add up to

$$
\begin{aligned}
& \frac{2^{5}}{4}\left[\sqrt{v_{4}}\left(1-\frac{n_{N}}{32}\right)+\frac{1}{\sqrt{v_{4}}}\left(1-\frac{n_{D 1}+n_{D 2}}{32}\right)\right]^{2} V_{4} I_{4}\left(I_{4} I_{4}\right)_{B} \\
+ & \frac{2^{5}}{4}\left[\sqrt{v_{4}}\left(1-\frac{n_{N}}{32}\right)+-\frac{1}{\sqrt{v_{4}}}\left(1-\frac{n_{D 1}+n_{D 2}}{32}\right)\right]^{2} I_{4} V_{4}\left(I_{4} I_{4}\right)_{B} \\
- & \frac{2^{5}}{4}\left[\sqrt{v_{4}}\left(1-\frac{n_{N}}{32}\right)+\frac{n_{D 1}-n_{D 2}}{\sqrt{v_{4}}}\right]^{2} S_{4} S_{4}\left(I_{4} I_{4}\right)_{B} \\
- & \frac{2^{5}}{4}\left[\sqrt{v_{4}}\left(1-\frac{n_{N}}{32}\right)+\frac{n_{D 2}-n_{D 1}}{\sqrt{v_{4}}}\right]^{2} S_{4} S_{4}\left(I_{4} I_{4}\right)_{B} .
\end{aligned}
$$

One thus finds

$$
n_{N}=32, \quad n_{D_{1}}=16 \quad n_{D_{2}}=16
$$

In view of (7.19), the $Z_{2}$ splittings of the Chan-Paton charge spaces can describe "complex" charges associated to unitary groups, as in eq. (7.14). Thus, letting

$$
\begin{array}{ll}
n_{N}=n+\bar{n}, & R_{N}=i(n-\bar{n}), \\
n_{D_{1}}=m_{1}+\bar{m}_{1}, & n_{D_{2}}=m_{2}+\bar{m}_{2}, \\
R_{D_{1}}=i\left(m_{1}-\bar{m}_{1}\right), & R_{D_{2}}=i\left(m_{2}-\bar{m}_{2}\right),
\end{array}
$$


one finds $n=16, m_{1}=8, m_{2}=8$, and the resulting gauge group is $U(8)_{5} \times U(8)_{5}^{\prime} \times U(16)_{9}$, where the two $U(8)$ factors originate from the D5 branes.

The massless open-string spectrum can be read from the relevant parts of eqs. (7.12) and (7.18), that are

$$
\begin{aligned}
\mathcal{A} & =n \bar{n} V_{4} I_{4}+m_{1} \bar{m}_{1}\left(V_{4} I_{4}-S_{4} S_{4}\right)+m_{2} \bar{m}_{2}\left(V_{4} I_{4}-C_{4} C_{4}\right) \\
& +\frac{n^{2}+\bar{n}^{2}}{2} I_{4} V_{4}+\frac{m_{1}^{2}+\bar{m}_{1}^{2}}{2}\left(I_{4} V_{4}-C_{4} C_{4}\right)+\frac{m_{2}^{2}+\bar{m}_{2}^{2}}{2}\left(I_{4} V_{4}-S_{4} S_{4}\right) \\
& +\left(n \bar{m}_{1}+\bar{n} m_{1}\right) Q_{S}^{\prime}+\left(n \bar{m}_{2}+\bar{n} m_{2}\right) Q_{S} \\
\mathcal{M} & =-\frac{n+\bar{n}}{2} \hat{I}_{4} \hat{V}_{4}-\frac{m_{1}+\bar{m}_{1}}{2}\left(\hat{I}_{4} \hat{V}_{4}-\hat{C}_{4} \hat{C}_{4}\right)-\frac{m_{2}+\bar{m}_{2}}{2}\left(\hat{I}_{4} \hat{V}_{4}-\hat{S}_{4} \hat{S}_{4}\right)
\end{aligned}
$$

Thus, in the $99(\mathrm{NN})$ sector, aside from the gauge bosons of $U(16)_{9}$, there are quartets of scalars in the $\mathbf{1 2 0}+\overline{\mathbf{1 2 0}}$ representations, while the corresponding fermionic modes, massless in the supersymmetric $U(16) \times U(16)$ model, are now massive as a result of supersymmetry breaking. In the 55 (DD) sector, aside from the gauge bosons of $U(8)_{5} \times U(8)_{5}^{\prime}$ and the corresponding adjoint fermions, $(\mathbf{6 4}, \mathbf{1})_{R}$ and $(\mathbf{1}, \mathbf{6 4})_{L}$, there are quartets of scalars and corresponding fermions in the representations $(\mathbf{2 8}, \mathbf{1})_{L},(\overline{\mathbf{2 8}}, \mathbf{1})_{L},(\mathbf{1}, \mathbf{2 8})_{R}$ and $(\mathbf{1}, \overline{\mathbf{2 8}})_{R}$. Finally, the states of the 59 (ND) sector are in mixed representations of the full gauge group $U(8)_{5} \times U(8)_{5}^{\prime} \times U(16)_{9}$. The corresponding massless spectrum consists of pairs of scalars and corresponding (half)fermions

Notice that, in analogy with the twisted sector of the closed string, the 55 and 59 sectors of the open spectrum contain even numbers of fermionic and bosonic modes at the massless level. This is just the phenomenon described in Section 5, since the 5-brane world-volume is orthogonal to the coordinate that breaks supersymmetry. As we already emphasized, however, there is a change of chirality for all fermions charged under the $U(8)_{5}^{\prime}$ gauge group, that reflects the corresponding phenomenon in the closed-string sector.

Finally, using the results of [22], one can compute the anomaly polynomial, that clearly

\footnotetext{
${ }^{11}$ This peculiar feature reflects the pesudoreality of six-dimensional Weyl spinors.
} 
contains no irreducible $R^{4}$ term, since there is no net number of chiral fermions or tensors. Moreover, the tadpole conditions eliminate the irreducible $F^{4}$ terms, and the residual anomaly polynomial,

$$
A=\frac{1}{4}\left(\operatorname{tr} F_{5}^{2}-\operatorname{tr} F_{5^{\prime}}^{2}\right)\left(\operatorname{tr} F_{9}^{2}+\frac{1}{2} \operatorname{tr} R^{2}\right)
$$

is rather similar to the corresponding one of the supersymmetric $U(16) \times U(16)$ model.

\section{Conclusions}

In this paper we have studied supersymmetry breaking by compactification in open descendants of the type IIB closed string theory, using a generalization to superstrings of the Scherk-Schwarz mechanism. We have exhibited two basic ways of realizing the breaking, that for the type IIB parent models originate from shifts of momenta and windings, respectively. The corresponding type-I descendants exhibit vastly different properties: in the former case the result is a rather direct extension to the open sector of the usual mechanism, while in the latter the massless open modes are not affected. Moreover, at tree level the first mechanism gives supersymmetry breaking masses of order $1 / R$ both in the closed and in the open sector. This is the case, since the direction used to implement the Scherk-Schwarz deformation is parallel to the worldvolume of the open-string branes. The corresponding models can be viewed as discrete deformations of supersymmetric ones containing Wilson lines, and are perturbatively dual to heterotic models with appropriate Scherk-Schwarz breaking. This correspondence was anticipated by duality arguments in Section 3.

In the second, qualitatively different mechanism, the coordinate used to implement the breaking is orthogonal to the worldvolume of the open-string branes, and in the closed sector the resulting soft masses are of order $R / \alpha^{\prime}$. The duality arguments of Section 3 associate this breaking to the eleventh dimension of $\mathrm{M}$ theory, and suggest that the massless open spectrum should not be affected at tree level. This is indeed confirmed by the explicit 
construction of Section 5. On the other hand, the massive open spectrum is affected by the breaking, that at one-loop is communicated to the massless states. The corresponding corrections, however, are exponentially suppressed in the radius as $e^{-1 / R}$. Therefore, the primary source of breaking in the open string massless spectrum are the gravitational interactions, expected to generate soft masses $\lesssim \mathcal{O}\left(m_{3 / 2}^{2} / M_{P}\right)$.

Type I models compactified to six and lower dimensions generically contain 9-branes and 5-branes. Since a generic compact coordinate is only parallel to the worldvolume of some of the branes, both mechanisms are expected to play a role. As we have seen, supersymmetry breaking along this coordinate affects at tree-level both the closed and the open spectrum of the branes parallel to it. On the other hand, the massless open spectra of branes orthogonal to this coordinate and from mixed sectors feel the breaking only through radiative corrections. When the corrections are purely of gravitational origin, the scale of supersymmetry breaking in the "orthogonal" branes is highly suppressed. Thus, one can attain phenomenologically interesting models with intermediate values for the compactification scale, of order $10^{12}-10^{14} \mathrm{GeV}$. Furthermore, the resulting scenario is compatible with gauge-coupling unification at the grand unified scale, identified also with the string scale $M_{I} \sim 10^{16} \mathrm{GeV}$. We emphasize that this mechanism is nonperturbative from the heterotic string point of view. It is certainly important to extend this construction to chiral four dimensional type I models [23], and to study its implications.

\section{Acknowledgements}

It is a pleasure to acknowledge stimulating discussions with C. Angelantonj, E. Kiritsis, C. Kounnas and H. Partouche. E.D. and A.S. would like to thank the Centre de Physique Théorique of the Ecole Polytechnique and the Laboratoire de Physique Théorique of the Ecole Normale Supérieure. A.S. would like to thank the Theory Division of CERN for the kind hospitality while this work was being completed, and M. Bianchi, G. Pradisi and Ya.S. Stanev, with whom the factorized form of the vacuum amplitudes was exhibited long ago 
in unpublished work.

\section{References}

[1] I. Antoniadis, Phys. Lett. B246 (1990) 377;

Proc. PASCOS-91 Symposium, Boston (World Scientific, Singapore, 1991), p. 718;

K. Benakli, Phys. Lett. B386 (1996) 106.

[2] I. Antoniadis, C. Munoz and M. Quiros, Nucl. Phys. B397 (1993) 515;

I. Antoniadis and K. Benakli, Phys. Lett. B326 (1994) 69;

I. Antoniadis, K. Benakli and M. Quirós, Phys. Lett. B331 (1994) 313;

I. Antoniadis, S. Dimopoulos and G. Dvali, Nucl. Phys. B516 (1998) 70.

[3] J. Scherk and J.H. Schwarz, Nucl. Phys. B153 (1979) 61, Phys. Lett. B82 (1979) 60;

E. Cremmer, J. Scherk and J.H. Schwarz, Phys. Lett. B84 (1979) 83;

P. Fayet, Phys. Lett. B159 (1985) 121, Nucl. Phys. B263 (1986) 649.

[4] R. Rohm, Nucl. Phys. B237 (1984) 553.

[5] C. Kounnas and M. Porrati, Nucl. Phys. B310 (1988) 355;

S. Ferrara, C. Kounnas, M. Porrati and F. Zwirner, Nucl. Phys. B318 (1989) 75;

C. Kounnas and B. Rostand, Nucl. Phys. B341 (1990) 641;

I. Antoniadis and C. Kounnas, Phys. Lett. B261 (1991) 369;

E. Kiritsis and C. Kounnas, Nucl. Phys. B503 (1997) 117.

[6] J.-P. Derendinger, L.E. Ibáñez and H.P. Nilles, Phys. Lett. B155 (1985) 65;

M. Dine, R. Rohm, N. Seiberg and E. Witten, Phys. Lett. B156 (1985) 55.

[7] A. Sagnotti, in: Cargese '87, Non-Perturbative Quantum Field Theory, eds. G. Mack et al. (Pergamon Press, Oxford, 1988) p. 521. 
[8] E. Witten, Nucl. Phys. B443 (1995) 85, B471 (1996) 135.

[9] J. Polchinski and E. Witten, Nucl. Phys. B460 (1996) 525.

[10] P. Horava and E. Witten, Nucl. Phys. B460 (1996) 506, B475 (1996) 94.

[11] I. Antoniadis and M. Quirós, Phys. Lett. B392 (1997) 61, Nucl. Phys. B505 (1997) 109, Phys. Lett. B416 (1998) 327.

[12] E. Dudas and C. Grojean, Nucl. Phys. B507 (1997) 553;

E. Dudas, Phys. Lett. B416 (1998) 309.

[13] C. Bachas, hep-th/9503030;

J.G. Russo and A.A. Tseytlin, Nucl. Phys. B461 (1996) 131, hep-th/9804076.

[14] G. Pradisi and A. Sagnotti, Phys. Lett. B216 (1989) 59.

[15] M. Bianchi and A. Sagnotti, Phys. Lett. B247 (1990) 517, Nucl. Phys. B361 (1991) 519.

[16] G. Pradisi, A. Sagnotti and Ya.S. Stanev, Phys. Lett. B354 (1995) 279, B356 (1995) 230, B381 (1996) 97;

J. Fuchs and C.Schweigert, Phys. Lett. B414 (1997) 251.

[17] M. Bianchi, G. Pradisi and A. Sagnotti, Nucl. Phys. B376 (1992) 365.

[18] J. Blum and K. Dienes, Phys. Lett. B414 (1997) 260, Nucl. Phys. B516 (1998) 83.

[19] K.S. Narain, Phys. Lett. B169 (1986) 41;

K.S. Narain, M.H. Sarmadi and E. Witten, Nucl. Phys. B279 (1987) 369.

[20] E. Gimon and J. Polchinski, hep-th/9601038.

[21] A. Dabholkar and J. Park, Nucl. Phys. B472 (1996) 207, B477 (1996) 701;

E. Gimon and C.V. Johnson, Nucl. Phys. B477 (1996) 715; 
M. Berkooz, R. Leigh, J. Polchinski, J.H. Schwarz, N. Seiberg and E. Witten, Nucl. Phys. B475 (1996) 115;

J. Blum and A. Zaffaroni, Phys. Lett. B387 (1996) 71;

J. Blum, Nucl. Phys. B486 (1997) 34;

A. Sen, Nucl. Phys. B475 (1996) 562,

Phys. Rev. D55 (1997) 7345, hep-th/9709159;

J. Blum and K. Intriligator, Nucl. Phys. B506 (1997) 199, B506 (1997) 223;

K. Dasgupta and S. Mukhi, Phys. Lett. B385 (1996) 125;

C. Angelantonj, M. Bianchi, G. Pradisi, A. Sagnotti and Ya.S. Stanev, Phys. Lett. B387 (1996) 743;

Z. Kakushadze, G. Shiu and S.-H. Tye, hep-th/9803141.

[22] L. Alvarez-Gaumé and E. Witten, Nucl. Phys. B234 (1984) 269.

[23] C. Angelantonj, M. Bianchi, G. Pradisi, A. Sagnotti and Ya. S. Stanev, Phys. Lett. B385 (1996) 96;

Z. Kakushadze, Nucl. Phys. B512 (1998) 221;

Z. Kakushadze and G. Shiu, Phys. Rev. D56 (1997) 3686, Nucl. Phys. B520 (1998) 75;

M. Bianchi, G. Pradisi and A. Sagnotti, 1991, as reported in:

A. Sagnotti, hep-th/9302099;

G. Zwart, hep-th/9708040;

G. Aldazabal, A. Font, L.E. Ibáñez and G. Violero, hep-th/9804026;

Z. Kakushadze, G. Shiu and S.-H.H. Tye, hep-th/9804092. 